\title{
Synthesis, spectroscopy, and theoretical calculations for a series of push- pull [14]-pyridoannulenes
}

Matthew G. Lauer, James W. Leslie, Ashley Mynar, Shelly A. Stamper, Anthony D. Martinez, Adrian J. Bray, Senai Negassi, Kevin McDonald, Eric Ferraris, Aaron Muzny, Shawn McAvoy, Chris Miller, Keith A. Walters* and Keith C. Russell*

Northern Kentucky University, Department of Chemistry, Highland Heights, KY 41099 - 1905 Evan Wang, Betsy Nuez and Carol Parish*

University of Richmond, Department of Chemistry, 28 Westhampton Way, Richmond, VA 23173

Experimental (Page S-2)

\begin{abstract}
Absorption and Emission Spectra
Absorption spectra of (a) 13, (b) 16, and (c) 19. Spectra are recorded in $\mathrm{CH}_{2} \mathrm{Cl}_{2}$. (Page S-9) Absorption spectra of (a) 5, (b) 6, and (c) 7. Spectra are recorded in $\mathrm{CH}_{2} \mathrm{Cl}_{2}$. (Page S-10) Emission spectra and TFA titration of (a) 13, (b) 16, and (c) 19 recorded in $\mathrm{CH}_{2} \mathrm{Cl}_{2}$. (Page S-11) Emission spectra and TFA titration of (a) 5, (b) 6, and (c) 7. Spectra are recorded in $\mathrm{CH}_{2} \mathrm{Cl}_{2}$. (Page S-12)
\end{abstract}

\section{Computational data}

Table 3. Energetics of 5' - 7' calculated at the B3LYP/6-31G* level of theory. (Page S-13) Cartesian coordinates, number of imaginary frequencies and energy for 5' (Page S-14) Cartesian coordinates, number of imaginary frequencies and energy for 6' (Page S-15) Cartesian coordinates, number of imaginary frequencies and energy for 7' (Page S-16) Cartesian coordinates, number of imaginary frequencies and energy for 13' (Page S-17) Cartesian coordinates, number of imaginary frequencies and energy for $16^{\prime}$ (Page S-18) Cartesian coordinates, number of imaginary frequencies and energy for 19' (Page S-19) Cartesian coordinates, number of imaginary frequencies and energy for 21 (Page S-20) Cartesian coordinates, number of imaginary frequencies and energy for 22 (Page S-21) Cartesian coordinates, number of imaginary frequencies and energy for 23 (Page S-22) Cartesian coordinates, number of imaginary frequencies and energy for $5^{\prime} \cdot \mathrm{H}($ Page S-23) Cartesian coordinates, number of imaginary frequencies and energy for $6^{\prime} \cdot \mathrm{H}$ (Page S-24) Cartesian coordinates, number of imaginary frequencies and energy for $7^{\prime} \cdot \mathrm{H}($ Page S-25) Cartesian coordinates, number of imaginary frequencies and energy for $7^{\prime} \cdot \mathrm{H}^{\prime}$ (Page S-26) Cartesian coordinates, number of imaginary frequencies and energy for $5^{\prime} \cdot 2 \mathrm{H}$ (Page S-27) Cartesian coordinates, number of imaginary frequencies and energy for $6^{\prime} \cdot 2 \mathrm{H}$ (Page S-28) Cartesian coordinates, number of imaginary frequencies and energy for $7^{\prime} \cdot 2 \mathrm{H}$ (Page S-29) 


\section{Selected Experimentals}

\section{General}

All reagents and catalysts were unless otherwise noted were used as received. Diisopropylamine used in palladium catalyzed coupling reactions was stored over $4 \AA$ molecular sieves and bubbled with argon for a minimum of 30 min using a gas dispersion tube prior to use. ${ }^{1} \mathrm{H}$ and ${ }^{13} \mathrm{C}$ NMR spectra were obtained at field strengths of $500 \mathrm{MHz}$ and $126 \mathrm{MHz}$, respectively. Chemical shifts are recorded in parts per million on the $\delta$ scale referenced to the solvent peak as an internal standard. Melting points obtained are uncorrected. UV-Vis spectra were obtained using a scanning spectrophotometer. Excitation of the samples for emission spectra was into the most intense absorption band in all cases.

3-Triflic 2-triisopropylsilyethynylpyridinate. Into a $75 \mathrm{~mL}$ pressure tube was placed 3-triflic 2bromopyridinate ( $2.513 \mathrm{~g}, 8.210 \mathrm{mmol})$, a stir bar, and degassed diisopropylamine ( 43 $\mathrm{mL})$. The mixture was bubbled with $\mathrm{Ar}$ and $\mathrm{Pd}\left(\mathrm{PhCN}_{2}\right)_{2} \mathrm{Cl}_{2}(0.094 \mathrm{~g}, 3 \mathrm{~mol} \%), \mathrm{CuI}(0.044 \mathrm{~g}, 2 \mathrm{~mol} \%)$, and $\left[\left(\mathrm{t}-\mathrm{Bu}_{3}\right) \mathrm{PH}\right] \mathrm{BF}_{4}(0.142 \mathrm{~g}, 6 \mathrm{~mol} \%)$ were added. The mixture was bubbled for an additional five min and (triisopropylsilyl)-acetylene $(2.38 \mathrm{~mL}, 10.7 \mathrm{mmol})$ was added. The tube was capped and stirred at $\mathrm{rt}$ for $2 \mathrm{~h}$. The tube was then heated to $65^{\circ} \mathrm{C}$ for two days. After cooling to room temperature, the reaction mixture was evaporated to dryness by rotary evaporation under reduced pressure and subjected to flash chromatography in $0-1 \%$ EtOAc/Hexanes. The product, $2.40 \mathrm{~g}$, was

obtained as a light brown oil in a yield of $72 \% .{ }^{1} \mathrm{H}$ NMR $\left(\mathrm{CDCl}_{3}\right) \delta 8.60(\mathrm{~d}, \mathrm{~J}=4.6 \mathrm{~Hz}, 1 \mathrm{H}), 7.64$ $(\mathrm{d}, \mathrm{J}=8.3 \mathrm{~Hz}, 1 \mathrm{H}), 7.34(\mathrm{dd}, \mathrm{J}=4.6 \mathrm{~Hz}, 8.3 \mathrm{~Hz}, 1 \mathrm{H}), 1.18(\mathrm{~m}, 21 \mathrm{H}) ;{ }^{13} \mathrm{C} \mathrm{NMR}\left(\mathrm{CDCl}_{3}\right) \delta$ 149.3, 147.9, 137.6, 129.0, 123.9, 118.7 (q, C-F coupling, $\mathrm{J}=321 \mathrm{~Hz}), 101.3,99.2,18.6,11.2 ; \mathrm{IR}(\mathrm{KBr}) v$ 3067, 2949, 2868, 2164, 1594, 1438, $1218 \mathrm{~cm}^{-1}$; ESI-HRMS calc'd for $\mathrm{C}_{17} \mathrm{H}_{24} \mathrm{~F}_{3} \mathrm{NO}_{3} \mathrm{SSi}$ : 430.1096 $[\mathrm{M}+\mathrm{Na}]^{+}$, found: 430.1098. 
2-Triisopropylsilylethynyl-3-trimethysilylethynylpyridine (9). Into a $48 \mathrm{~mL}$ pressure tube was placed 3-Triflic 2-triisopropylsilyethynyl pyridinate $(2.96 \mathrm{~g}, 7.26 \mathrm{mmol})$, a stir bar, and degassed diisopropylamine $(\sim 40 \mathrm{~mL})$. The solution was bubbled with $\mathrm{Ar}$ and $\mathrm{Pd}\left(\mathrm{PPh}_{3}\right)_{4}(0.280 \mathrm{~g}, 3 \mathrm{~mol} \%)$ was added followed by the addition of trimethysilyl-acetylene (1.96 mL, $13.9 \mathrm{mmol})$. Additional degassed diisopropylamine was also added to leave room for expansion of the solvent, but to minimize the headroom once the reaction reached temperature. The mixture was heated to $120{ }^{\circ} \mathrm{C}$ behind a blast shield and stirred over-night. The reaction mixture was cooled to rt and evaporated to dryness by rotary evaporation under reduced pressure. The residue was subjected to flash chromatography in $0.25 \%$ EtOAc/Hexanes. The title compound, a yellow oil, was obtained in a yield of $72 \%(1.81 \mathrm{~g}) .{ }^{1} \mathrm{H} \mathrm{NMR}\left(\mathrm{CDCl}_{3}\right) \delta 8.50(\mathrm{~d}, \mathrm{~J}=4.1 \mathrm{~Hz}, 1 \mathrm{H}), 7.76(\mathrm{~d}, \mathrm{~J}=7.8 \mathrm{~Hz}, 1 \mathrm{H}), 7.17$ $(\mathrm{dd}, \mathrm{J}=4.6 \mathrm{~Hz}, 7.8 \mathrm{~Hz}, 1 \mathrm{H}), 1.16(\mathrm{~m}, 21 \mathrm{H}), 0.24(\mathrm{~s}, 9 \mathrm{H}) ;{ }^{13} \mathrm{C} \mathrm{NMR}\left(\mathrm{CDCl}_{3}\right) \delta 148.8,144.4,140.3$, $122.9,122.0,104.3,101.7,101.0,96.0,18.9,11.3,-0.1$; IR (KBr) v 3037, 2945, 2866, 2165, 1573, 1552, 1463, $1415 \mathrm{~cm}^{-1}$; ESI-HRMS calc'd for $\mathrm{C}_{21} \mathrm{H}_{33} \mathrm{NSi}_{2}: 356.2230[\mathrm{M}+\mathrm{H}]^{+}$, found: 356.2229 .

3-Triflic 2-trimethysilylethynylpyridinate. Into a $75 \mathrm{~mL}$ pressure tube was placed 3-triflic 2bromopyridinate $(4.016 \mathrm{~g}, 13.12 \mathrm{mmol})$, a stir bar, and degassed diisopropylamine $(\sim 43 \mathrm{~mL})$. The solution was bubbled with argon and $\mathrm{Pd}(\mathrm{PhCN})_{2} \mathrm{Cl}_{2}(0.031 \mathrm{~g} ; 3 \mathrm{~mol} \%), \mathrm{CuI}(0.051 \mathrm{~g} ; 2 \mathrm{~mol} \%)$, and $\left[\left(\mathrm{t}-\mathrm{Bu}_{3}\right) \mathrm{PH} \mathrm{BF}_{4}(0.228 \mathrm{~g}: 6 \mathrm{~mol} \%)\right.$ were added. The mixture was bubbled for an additional 5 min and trimethysilyl-acetylene $(2.41 \mathrm{~mL} ; 17.1 \mathrm{mmol})$ was added. The tube was capped, stirred at $\mathrm{rt}$ for $4 \mathrm{~h}$, and then heated to $58{ }^{\circ} \mathrm{C}$ for two days. The reaction mixture was evaporated to dryness by rotary evaporation under reduced pressure and subjected to flash chromatography in $0-1 \%$ EtOAc/Hexanes. The product, a light brown solid (3.56 g), was obtained in $84 \%$ yield. $\mathrm{mp}=46.9$ $47.8{ }^{\circ} \mathrm{C} .{ }^{1} \mathrm{H} \mathrm{NMR}\left(\mathrm{CDCl}_{3}\right) \delta 8.58(\mathrm{~d}, \mathrm{~J}=3.7 \mathrm{~Hz}, 1 \mathrm{H}), 7.62(\mathrm{~d}, \mathrm{~J}=8.7,1 \mathrm{H}), 7.36(\mathrm{dd}, \mathrm{J}=4.6 \mathrm{~Hz}$, $8.3 \mathrm{~Hz}, 1 \mathrm{H}), 0.28(\mathrm{~s}, 9 \mathrm{H}) ;{ }^{13} \mathrm{C} \mathrm{NMR}\left(\mathrm{CDCl}_{3}\right) \delta 149.3,147.9,137.3,129.3,124.1,118.7$ (q, C-F 
coupling, $\mathrm{J}=321 \mathrm{~Hz}), 104.3,97.0,-0.7$; IR $(\mathrm{KBr}) \vee 3075,2970,2160,1586,1438,1231 \mathrm{~cm}^{-1}$; ESIHRMS calc'd for $\mathrm{C}_{11} \mathrm{H}_{12} \mathrm{~F}_{3} \mathrm{NO}_{3} \mathrm{SSi}: 324.0337[\mathrm{M}+\mathrm{H}]^{+}$, found: 324.0343 .

3-triisopropylsilylethynyl-2-trimethysilylethynylpyridine (10). Into a $75 \mathrm{~mL}$ pressure tube was placed 3-Triflic 2-trimethysilylethynylpyridinate (2.46 g; $7.61 \mathrm{mmol})$, a stir bar, and degassed diisopropylamine $(\sim 45 \mathrm{~mL})$. The solution was bubbled with argon for five min before $\operatorname{Pd}\left(\mathrm{PPh}_{3}\right)_{4}$ $(0.264 \mathrm{~g} ; 3 \mathrm{~mol} \%)$ was added. The mixture bubbled an additional three minutes and triisopropylsilyl-acetylene $(1.8 \mathrm{~mL} ; 8.1 \mathrm{mmol})$ was added. Additional degassed diisopropylamine was also added to leave room for expansion of the solvent to to minimize the headroom once the reaction reaced temperature. The tube was capped, the mixture was heated to $120{ }^{\circ} \mathrm{C}$ behind a blast shield, and stirred over-night. After cooling to room temperature, the reaction mixture was evaporated to dryness by rotary evaporation under reduced pressure and subjected to flash chromatography in $0-1 \%$ EtOAc/Hexanes. The product was a red oil, obtained in a yield of $90 \%$ $(2.44 \mathrm{~g})$. The spectral characteristics of the product were identical to those previously reported. ${ }^{26}$

2-Ethynyl-3-triisopropylsilylethynylpyridine (11). In a $250 \mathrm{~mL}$ Erlenmeyer flask was placed $\mathrm{ca}$. $160 \mathrm{~mL}$ of sat. $\mathrm{K}_{2} \mathrm{CO}_{3} / \mathrm{MeOH}$ solution, $9(0.800 \mathrm{~g}, 2.25 \mathrm{mmol})$, and a large stir bar. A septum was fitted to the flask and the solution was stirred at a high rate of speed for $24 \mathrm{~h}$ at $\mathrm{rt}$. The solution was then concentrated down to $c a .2-3 \mathrm{~mL}$ by rotary evaporation at reduced pressure on a $45^{\circ} \mathrm{C}$ water bath. The concentrate was diluted with ether $(80 \mathrm{~mL})$ and the ether solution was washed with water (4 x $50 \mathrm{~mL}$ ), dried over $\mathrm{MgSO}_{4}$, and the solvent removed by rotary evaporation on a $45^{\circ} \mathrm{C}$ water bath followed by high vacuum. The crude product (592 mg, 93\%) was obtained as a viscous, pale, brown oil. The crude product was sufficiently pure for use without any additional purification, however, a small sample was purified by column chromatography for analysis. ${ }^{1} \mathrm{H} \mathrm{NMR}\left(\mathrm{CDCl}_{3}\right)$ :

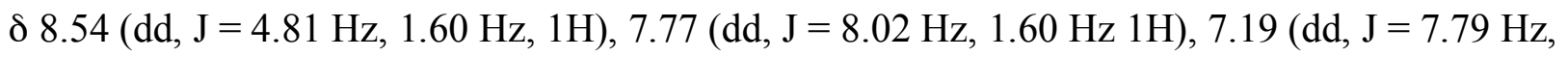


$5.04 \mathrm{~Hz}, 1 \mathrm{H}), 3.37(\mathrm{~s}, 1 \mathrm{H}), 1.16(\mathrm{~m}, 21 \mathrm{H}) ;{ }^{13} \mathrm{C} \mathrm{NMR}\left(\mathrm{CDCl}_{3}\right) \delta$ 149.2, 145.6, 139.8, 122.3, 122.1, 104.0, 96.5, 83.8, 80.2, 18.7, 11.3; IR (KBr) v 3201, 2945, 2860, 2104, 1549, 1461, $1415 \mathrm{~cm}^{-1}$; ESIHRMS calc'd for $\mathrm{C}_{18} \mathrm{H}_{26} \mathrm{NSi}: 284.1835[\mathrm{M}+\mathrm{H}]^{+}$, found: 284.1831 .

1,2-Dibutoxybenzene. In a $250 \mathrm{~mL}$ round-bottomed flask, catechol ( $8.8 \mathrm{~g}, 80 \mathrm{mmol})$ was dissolved in DMSO (30 mL) giving a slight yellow soultion. The solution was deoxygenated by three freeze, pump, thaw and fill cycles with argon. Separately, iodobutane $(40 \mathrm{~mL}, 350 \mathrm{mmol})$ was bubbled under argon for $10 \mathrm{~min}$. The idodobutane and potassium carbonate $(25 \mathrm{~g}, 181.4 \mathrm{mmol})$ was then added to the original solution. Finally, the mixture was stirred at $100{ }^{\circ} \mathrm{C}$ overnight under Ar. After the mixture was cooled to rt, the DMSO was removed by rotary evaporation under high vacuum. Water $(100 \mathrm{~mL})$ and methylene chloride $(40 \mathrm{~mL})$ were then added to the residue. The organic layer was set aside, and the aqueous layer was extracted with methylene chloride ( $3 \mathrm{X} 30 \mathrm{~mL})$. The combined organic solutions were washed with water $(2$ X $50 \mathrm{~mL})$, dried over anhyd. sodium sulfate and filtered. The volatiles were removed by rotary evaporation under reduced pressure. The resulting dark residue was purified by fractional distillation with the aid of a short Vigreaux column. The title compound was collected at $100-110{ }^{\circ} \mathrm{C}$ at 1 torr to yield $2.5 \mathrm{~g}$ of a light, yellowish oil (14\%). ${ }^{1} \mathrm{H}$ NMR $\left(\mathrm{CDCl}_{3}\right): \delta 6.88(\mathrm{~s}, 2 \mathrm{H}), 3.99(\mathrm{t}, 2 \mathrm{H}), 1.79(\mathrm{~m}, 2 \mathrm{H}), 1.51(\mathrm{~m}, 2 \mathrm{H})$, $0.97(\mathrm{t}, 3 \mathrm{H})$.

1,2-dibutoxy-4,5-diiodobenzene (12). Periodic acid (2.10 g, $9.21 \mathrm{mmol})$ was mixed with methanol $(12.3 \mathrm{~mL})$ in a $100 \mathrm{~mL}$ round-bottomed flask and stirred for 15 minutes at $20{ }^{\circ} \mathrm{C}$. Iodine $(2.0 \mathrm{~g}, 7.9$ mmol) was then added and the mixture stirred vigorously for ten minutes. Subsequently, 1,2dibutoxybenzene $(2.0 \mathrm{~g}, 9.0 \mathrm{mmol})$ was added and the mixture was stirred for $4 \mathrm{hr}$ at $70{ }^{\circ} \mathrm{C}$. It was noted that the color of the solution turned to dark brown with a dark precipitate. The hot mixture was then poured into $1 \% \mathrm{NaOH}(100 \mathrm{~mL})$ and the color of the solution turned to yellow with dark 
brown oily precipitate. The solution was decanted and the precipitate recrystallized from a small amount of methanol. The title compound was isolated as white crystals in $38 \%$ yield (1.62 g). mp: 33.5-34.5 ${ }^{\circ} \mathrm{C} .{ }^{1} \mathrm{H}$ NMR $\left(\mathrm{CDCl}_{3}\right): \delta 7.24(\mathrm{~s}, 2 \mathrm{H}), 3.92(\mathrm{t}, \mathrm{J}=6.9 \mathrm{~Hz}, 4 \mathrm{H}), 1.76(\mathrm{~m}, 4 \mathrm{H}), 1.96(\mathrm{~m}, 4 \mathrm{H})$, $0.96(\mathrm{t}, \mathrm{J}=7.3 \mathrm{~Hz}, 6 \mathrm{H}) ;{ }^{13} \mathrm{C} \mathrm{NMR}\left(\mathrm{CDCl}_{3}\right): 149.9,123.9,96.1,69.3,31.2,19.2,13.9$; IR $(\mathrm{KBr}) v$ 2954, 2927, 1494, 1463, $\mathrm{cm}^{-1}$; ESI-HRMS calc'd for $\mathrm{C}_{14} \mathrm{H}_{20} \mathrm{I}_{2} \mathrm{O}_{2}: 496.9445$ [M+Na] $]^{+}$, found: 496.9449.

3-Ethynyl-2-triisopropylsilylethynylpyridine (15). Prepared from 10 (2.00 g, 5.62 mmol), using the same procedure used for 11. The title compound was isolated in $90 \%$ yield $(1.43 \mathrm{~g})$. The spectral data of the product were consistent with that reported in the literature. ${ }^{26}$

Compound 16. In a $15 \mathrm{~mL}$ pressure tube was placed $c a .13 \mathrm{~mL}$ of degassed diisopropylamine, 15 (0.500 g, $1.76 \mathrm{mmol}), 12$ (0.398 g, $0.84 \mathrm{mmol}), \mathrm{Pd}\left(\mathrm{PPh}_{3}\right)_{2} \mathrm{Cl}_{2}(0.059 \mathrm{~g}, 10 \mathrm{~mol} \%), \mathrm{CuI}(0.016 \mathrm{~g}, 10$ mol \%), and a stir bar. The solution was bubbled with argon for 3 min., the tube purged with argon, and the cap quickly fitted. The tube was placed in an oil bath heated to $80{ }^{\circ} \mathrm{C}$ and left for 3 days with stirring. The reaction mixture was concentrated by rotary evaporation at reduced pressure on a $45^{\circ} \mathrm{C}$ bath followed by high vacuum. The crude product was purified by flash chromatography on silica gel eluted with 5 - 20\% EtOAc in hexanes. The product, $536 \mathrm{mg}$, was recovered as a thick, pale brown oil $(81 \%)$. The product ${ }^{1} \mathrm{H}$ NMR $\left(\mathrm{CDCl}_{3}\right): \delta 8.52(\mathrm{~d}, \mathrm{~J}=4.12 \mathrm{~Hz}, 2 \mathrm{H}), 7.75(\mathrm{dd}, \mathrm{J}=$ $8.02 \mathrm{~Hz}, 1.60 \mathrm{~Hz}, 2 \mathrm{H}), 7.15$ (dd, J = 7.79 Hz, 4.58 Hz, 2H), 7.04 (s, 2H), 3.99 (t, J = 6.87 Hz, 4H), $1.82(\mathrm{~m}, 4 \mathrm{H}), 1.49(\mathrm{~m} \mathrm{4H}), 1.07(\mathrm{~m}, 42 \mathrm{H}), 0.99(\mathrm{t}, \mathrm{J}=7.33 \mathrm{~Hz}, 6 \mathrm{H}) ;{ }^{13} \mathrm{C} \mathrm{NMR}\left(\mathrm{CDCl}_{3}\right) \delta 149.5$, $148.7,145.2,139.9,122.8,121.7,118.4,116.7,103.1,98.4,91.8,69.0,31.3,19.3,18.7,13.9,11.4$ ESI-HRMS calc'd for $\mathrm{C}_{50} \mathrm{H}_{68} \mathrm{~N}_{2} \mathrm{O}_{2} \mathrm{Si}_{2}$ : $785.4897[\mathrm{M}+\mathrm{H}]^{+}$, found: 785.4877 .

Compound 17. This compound was prepared in the same manner as 14. The protected starting material, $16(0.250 \mathrm{~g}, 0.318 \mathrm{mmol})$, yielded $83 \mathrm{mg}(55 \%)$ of mostly pure product after a short silica 
gel column using 10-25\% EtOAc in hexanes as the eluent. The product was used in the next reaction without further purification. ${ }^{1} \mathrm{H} \mathrm{NMR}\left(\mathrm{CDCl}_{3}\right): \delta 8.53(\mathrm{dd}, \mathrm{J}=4.58 \mathrm{~Hz}, 1.37 \mathrm{~Hz}, 2 \mathrm{H}), 7.75$ $(\mathrm{dd}, \mathrm{J}=8.02 \mathrm{~Hz}, 1.60 \mathrm{~Hz}, 2 \mathrm{H}), 7.16(\mathrm{~m}, 2 \mathrm{H}), 7.15(\mathrm{~s}, 2 \mathrm{H}), 4.01(\mathrm{t}, \mathrm{J}=6.87 \mathrm{~Hz}, 2 \mathrm{H}), 3.56(\mathrm{~s}, 2 \mathrm{H})$, $1.81(\mathrm{~m}, 4 \mathrm{H}), 1.49(\mathrm{~m} 4 \mathrm{H}), 0.97(\mathrm{t}, \mathrm{J}=7.33 \mathrm{~Hz}, 6 \mathrm{H})$.

Compound 18. In a $75 \mathrm{~mL}$ pressure tube was placed $c a .70 \mathrm{~mL}$ of degassed diisopropylamine, 15 (0.300 g, $1.06 \mathrm{mmol}), 12$ (1.01 g, $2.12 \mathrm{mmol}), \mathrm{Pd}\left(\mathrm{PPh}_{3}\right)_{2} \mathrm{Cl}_{2}(0.068 \mathrm{~g}, 10 \mathrm{~mol} \%), \mathrm{CuI}(0.018 \mathrm{~g}, 10$ mol \%), and a stir bar. The solution was bubbled with argon for 3 min., the tube purged with argon, and the cap quickly fitted. The tube was placed in an oil bath for 2 days at $\mathrm{rt}$. The reaction mixture was concentrated by rotary evaporation at reduced pressure on a $45{ }^{\circ} \mathrm{C}$ bath followed by high vacuum. The crude product was purified by flash chromatography on silica gel eluted with $5-10 \%$ EtOAc in hexanes. The product, $536 \mathrm{mg}$, was recovered as a pale brown oil $(50 \%, \mathrm{ca}$. $85 \%$ pure by ${ }^{1} \mathrm{H}$ NMR). The compound was used in the next reaction without futher purification

Compound 19. In a $15 \mathrm{~mL}$ pressure tube was placed ca. $13 \mathrm{~mL}$ of degassed diisopropylamine, partially purified $18(0.777 \mathrm{~g}, 1.23 \mathrm{mmol}), 11(0.400 \mathrm{~g}, 1.41 \mathrm{mmol}), \mathrm{Pd}\left(\mathrm{PPh}_{3}\right)_{2} \mathrm{Cl}_{2}(0.099 \mathrm{~g}, 10 \mathrm{~mol}$ $\%), \mathrm{CuI}(0.027 \mathrm{~g}, 10 \mathrm{~mol} \%)$, and a stir bar. The solution was bubbled with argon for $3 \mathrm{~min}$. , the tube purged with argon, and the cap quickly fitted. The tube was placed in an oil bath for 3 days at $80{ }^{\circ} \mathrm{C}$. The reaction mixture was concentrated by rotary evaporation at reduced pressure on a $45^{\circ} \mathrm{C}$ bath followed by high vacuum. The crude product was purified by flash chromatography on silica gel eluted with 5-7.5\% EtOAc in hexanes followed by a second column eluted with 3-5\% EtOAc in hexanes. The product was obtained as an viscous, tacky, brown oil in $66 \%$ yield $(640 \mathrm{mg}) .{ }^{1} \mathrm{H}$ $\operatorname{NMR}\left(\mathrm{CDCl}_{3}\right): \delta 8.53(\mathrm{~d}, \mathrm{~J}=4.1 \mathrm{~Hz}, 1 \mathrm{H}), 8.45(\mathrm{~d}, \mathrm{~J}=3.7 \mathrm{~Hz}, 1 \mathrm{H}), 8.14(\mathrm{~d}, \mathrm{~J}=7.8 \mathrm{~Hz}, 1 \mathrm{H}), 7.79$ $(\mathrm{d}, \mathrm{J}=7.8 \mathrm{~Hz}, 1 \mathrm{H}), 7.19(\mathrm{dd}, \mathrm{J}=7.8 \mathrm{~Hz}, 4.6 \mathrm{~Hz}, 1 \mathrm{H}), 7.12(\mathrm{dd}, \mathrm{J}=8.0 \mathrm{~Hz}, 4.6 \mathrm{~Hz}, 1 \mathrm{H}), 7.04(\mathrm{~s}$, 1H), $6.98(\mathrm{~s}, 1 \mathrm{H}), 3.99(\mathrm{~m}, 4 \mathrm{H}), 1.83(\mathrm{~m}, 4 \mathrm{H}), 1.51(\mathrm{~m} \mathrm{4H}), 1.17(\mathrm{~m}, 21 \mathrm{H}), 1.07(\mathrm{~m}, 21 \mathrm{H}), 0.99(\mathrm{~m}$, 
$6 \mathrm{H}) ;{ }^{13} \mathrm{C}$ NMR $\left(\mathrm{CDCl}_{3}\right) \delta 149.8,149.5,148.7,147.8,145.0,142.9,141.5,140.1,123.9,122.7$, $122.4,122.0,119.1,118.8,116.1,115.3,104.2,102.8,98.9,96.6,95.4,92.1,90.5,89.3,69.00$, 68.95, 31.32, 31.27, 19.3, 18.9, 18.7, 13.9, 11.42, 11.37; IR (KBr) v 2940, 2860, 2211, 2156, 1514, $1248 \mathrm{~cm}^{-1}$; ESI-HRMS calc'd for $\mathrm{C}_{50} \mathrm{H}_{68} \mathrm{~N}_{2} \mathrm{O}_{2} \mathrm{Si}_{2}: 785.4897[\mathrm{M}+\mathrm{H}]^{+}$, found: 785.4883.

Compound 20. In a $500 \mathrm{~mL}$ rb flask was placed $250 \mathrm{~mL}$ of THF, 19 (412 mg, $0.525 \mathrm{mmol})$, and a stir bar. A septum was fitted and two needles were inserted. One was connected to an argon line and a slight flow of argon through the flask was established. The solution was cooled to $-78^{\circ} \mathrm{C}$ and with vigorous stirring TBAF (1.58 mL, $1.58 \mathrm{mmol}, 1.0 \mathrm{M}$ in THF) was added dropwise. The solution was stirred for $1 \mathrm{~h}$ at $-78{ }^{\circ} \mathrm{C}$ then allowed to return to $\mathrm{rt}$. Water was added $(30 \mathrm{~mL})$ to quench the reaction and the reaction mixture was then concentrated by rotary evaporation at reduced pressure on a $45{ }^{\circ} \mathrm{C}$ water bath which removed most of the THF. The concentrate was rinsed into a separatory funnel with $c a .80 \mathrm{~mL}$ of ether and the ether solution was washed with water $(3 \times 50$ $\mathrm{mL}$ ), dried over $\mathrm{MgSO}_{4}$, and rotary evaporated under reduced pressure to give the crude product. The residue was passed over a short column of silica gel using 10-25\% EtOAc in hexanes as the eluent to yield $179 \mathrm{mg}$ (72\%, ca. $95 \%$ pure) of partially purified product which was used directly in the next reaction. ${ }^{1} \mathrm{H}$ NMR $\left(\mathrm{CDCl}_{3}\right): \delta 8.75(\mathrm{dd}, \mathrm{J}=4.8 \mathrm{~Hz}, 1.6 \mathrm{~Hz}, 1 \mathrm{H}), 8.50(\mathrm{dd}, \mathrm{J}=4.8 \mathrm{~Hz}, 1.6$ $\mathrm{Hz} 1 \mathrm{H}), 7.97$ (dd, J = 7.8 Hz, 1.4 Hz 1H), 7.81 (dd, J = 8.0 Hz, 1.6 Hz, 1H), 7.23 (m, 2H), 7.15 (s, 1H), $7.05(\mathrm{~s}, 1 \mathrm{H}), 4.04(\mathrm{~m}, 4 \mathrm{H}), 3.45(\mathrm{~s}, 1 \mathrm{H}), 3.20(\mathrm{~s}, 1 \mathrm{H}), 1.83(\mathrm{~m}, 4 \mathrm{H}), 1.51(\mathrm{~m} \mathrm{4H}), 0.99(\mathrm{~m}, 6 \mathrm{H})$. 


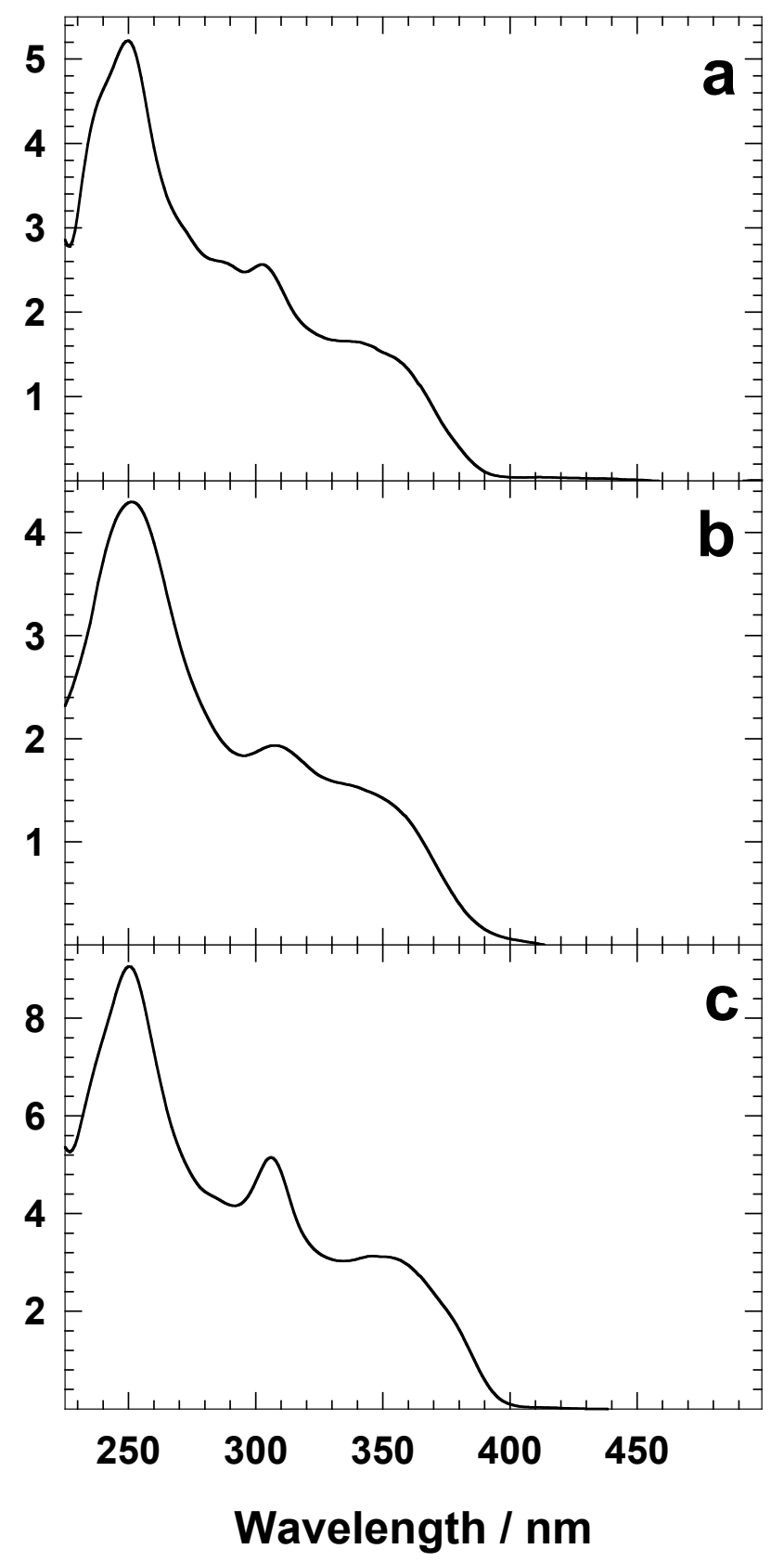

Absorption spectra of (a) 13, (b) 16, and (c) 19. Spectra are recorded in $\mathrm{CH}_{2} \mathrm{Cl}_{2}$. 


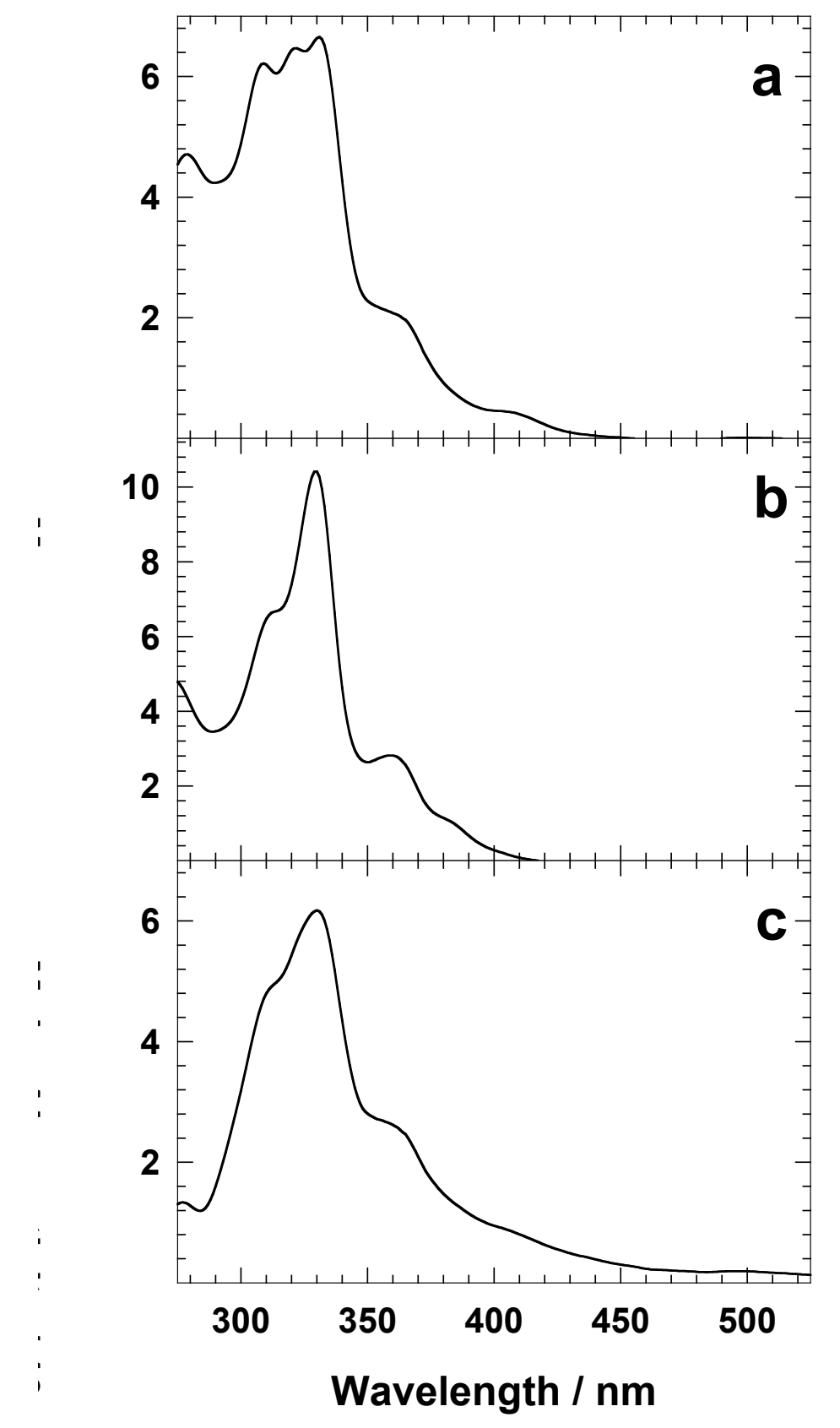

Absorption spectra of (a) 5, (b) 6, and (c) 7. Spectra are recorded in $\mathrm{CH}_{2} \mathrm{Cl}_{2}$. 


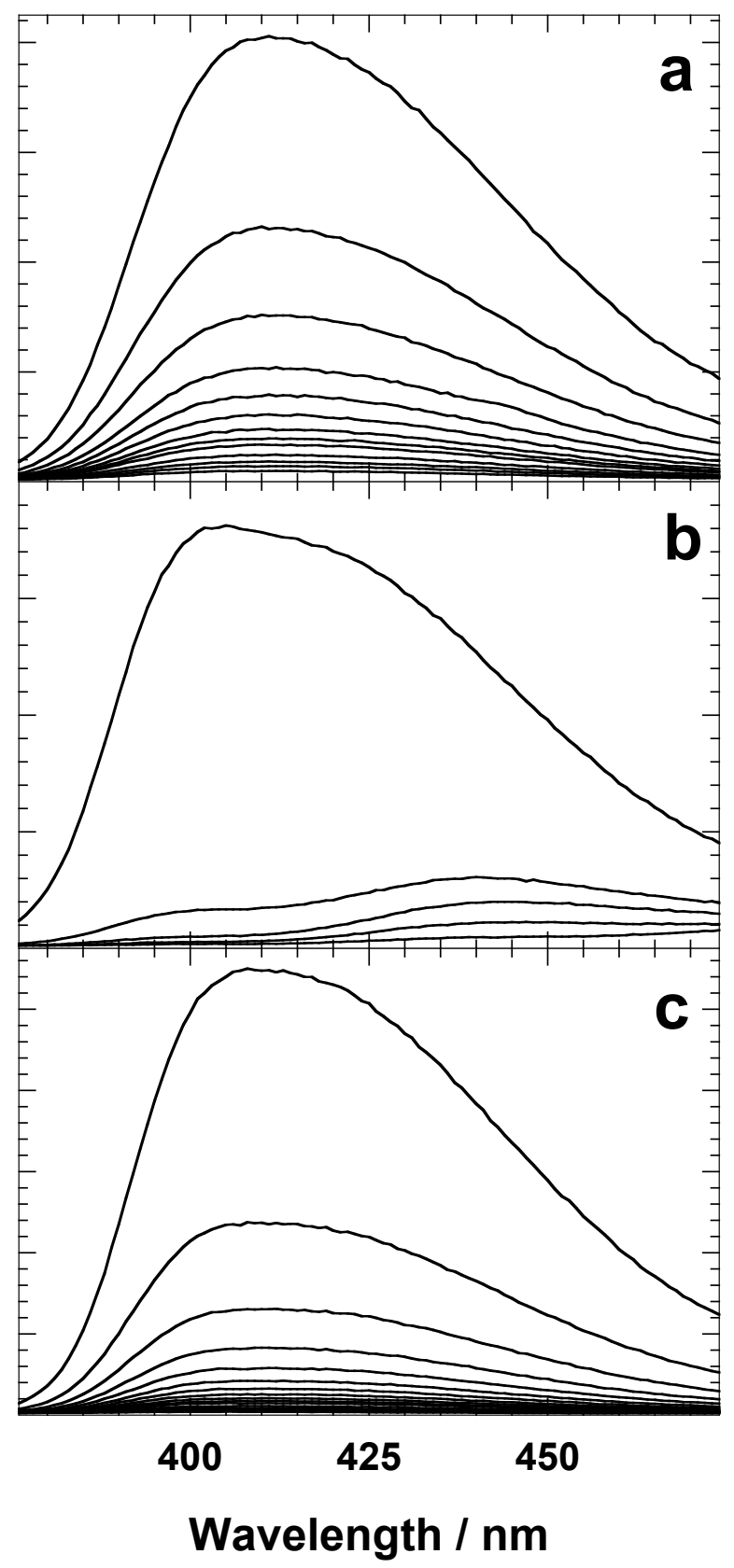

Emission spectra and TFA titration of (a) 13, (b) 16, and (c) 19. Spectra are recorded in $\mathrm{CH}_{2} \mathrm{Cl}_{2}$. Spectra are recorded after sequential aliquot addition ( 0.5 mol equiv.) of a methanol TFA solution. Emission intensity decreases with added TFA. 


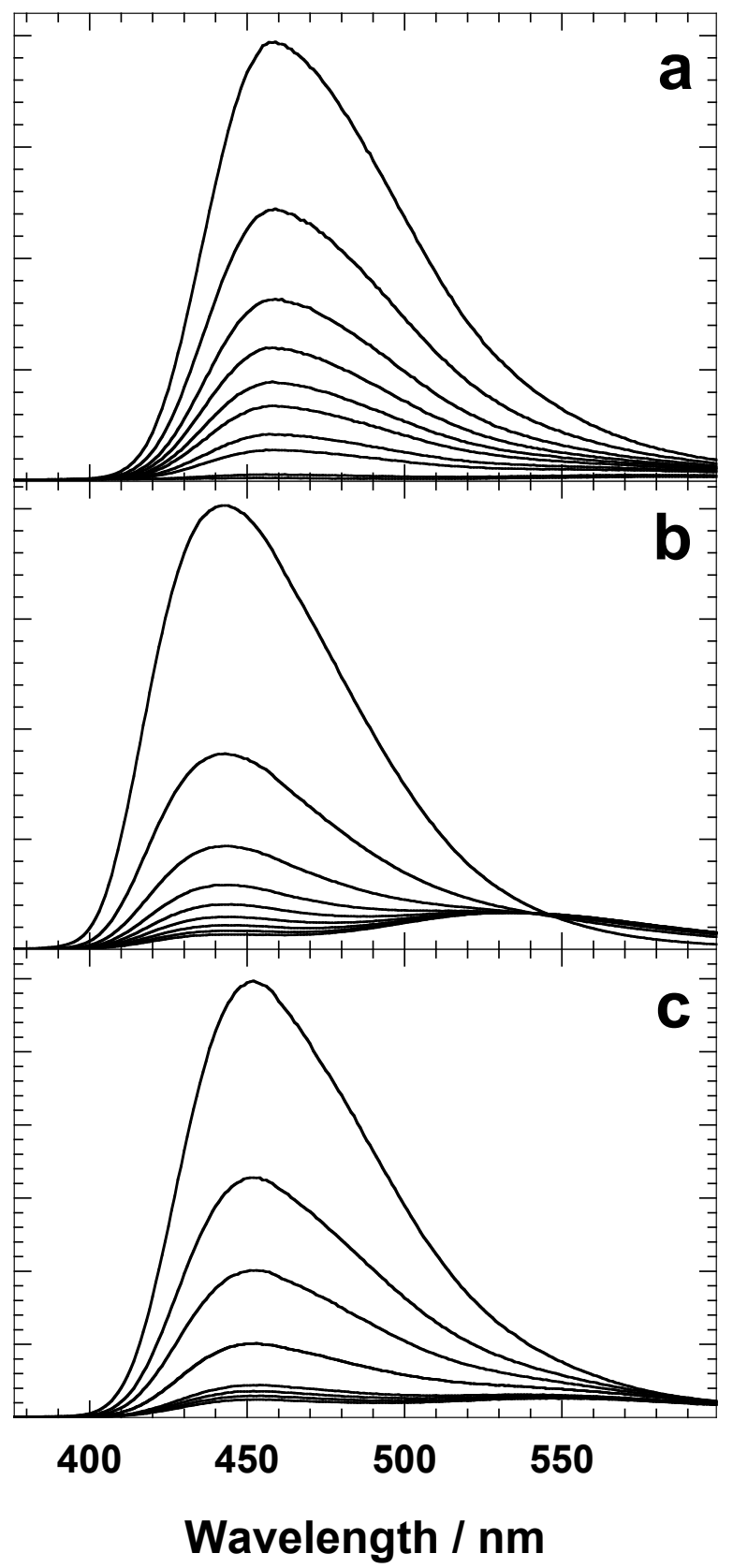

Emission spectra and TFA titration of (a) 5, (b) 6, and (c) 7. Spectra are recorded in $\mathrm{CH}_{2} \mathrm{Cl}_{2}$. Spectra are recorded after sequential aliquot addition ( 0.5 mol equiv.) of a methanol TFA solution. Emission intensity decreases with added TFA. 
Table 3. Energetics of $\mathbf{5}^{\prime}-\mathbf{7}^{\prime}$ calculated at the B3LYP/6-31G* level of theory.

\begin{tabular}{cccc}
\hline & Total Energy (a.u.) & $\Delta \mathrm{E}(\mathrm{kcal} / \mathrm{mol})$ & $\mu(\mathrm{D})$ \\
\hline $\mathbf{5}^{\prime}$ & -1258.88897 & 0.6705 & 6.8241 \\
$\mathbf{6}^{\prime}$ & -1258.89003 & 0.0000 & 0.5750 \\
$\mathbf{7}^{\prime}$ & -1258.88970 & 0.2077 & 3.1526
\end{tabular}


Cartesian coordinates, number of imaginary frequencies and energy for $\mathbf{5}^{\prime}$

Total energy: -1258.88897

Number of imaginary frequencies: 0

Cartesian coordinates:

\begin{tabular}{|c|c|c|c|}
\hline & 3.672544619919 & -0.045273300971 & \\
\hline & 3.664655460497 & -0.048415221746 & -0.959862120994 \\
\hline & 4.916378214811 & -0.065607659943 & -0.314856512431 \\
\hline$C$ & 6.077947620602 & -0.078855198103 & -1.072438523793 \\
\hline C & 5.967367275525 & -0.074550194832 & -2.468851184038 \\
\hline H & 4.953275617406 & -0.067909396834 & 0.770173917515 \\
\hline $\mathrm{H}$ & 7.054634891879 & -0.092112470824 & -0.598579779444 \\
\hline H & 6.860335083478 & -0.084684852329 & -3.091384186896 \\
\hline $\mathrm{C}$ & 1.470456485775 & -0.022691399652 & 0.503115943032 \\
\hline $\mathrm{C}$ & 2.465935342340 & -0.034678272076 & \\
\hline $\mathrm{C}$ & 1.252958989311 & -0.0111 & -3.404467757392 \\
\hline $\mathrm{C}$ & 2.424571768114 & -0.027475312163 & -3.061841773159 \\
\hline $\mathrm{C}$ & -1.300112899617 & 0.024353516630 & -3.682843030577 \\
\hline $\mathrm{C}$ & -0.08 & 343320010 & 1071 \\
\hline C & 605 & 0.07 & 7349 \\
\hline $\mathrm{C}$ & -4.843651438939 & 57424583 & -4.271333087593 \\
\hline $\mathrm{C}$ & & & \\
\hline C & -3.15 & & 96876997811 \\
\hline $\mathrm{C}$ & -4.5 & & 196 \\
\hline $\mathrm{H}$ & -6.47 & 0.09 & 7439 \\
\hline $\mathrm{H}$ & & & -5.149 \\
\hline $\mathrm{H}$ & -4.93 & 0.0681 & -0.878501998627 \\
\hline C & -1.3 & 0.0 & 6457 \\
\hline $\mathrm{C}$ & -1.8 & 0.0 & 5983 \\
\hline C & -0.935 & 0.0 & 1948 \\
\hline $\mathrm{C}$ & 0.470144077182 & -0.010592932792 & 1.51 \\
\hline C & & & \\
\hline $\mathrm{C}$ & 36 & -0.0 & \\
\hline $\mathrm{H}$ & -2.8 & 041 & 2540 \\
\hline $\mathrm{H}$ & 1.992 & -0.07 & 537987 \\
\hline $\mathrm{C}$ & -1.554185472627 & & -0.001137451678 \\
\hline $\mathrm{C}$ & & & \\
\hline $\mathrm{N}$ & 4.80 & -0.0 & 90418 \\
\hline $\mathrm{N}$ & -3.534166811734 & & -4.509522990394 \\
\hline $\mathrm{O}$ & 0.583797595893 & -0.034286431323 & 5.201822945322 \\
\hline $\mathrm{O}$ & & & 4.731174763440 \\
\hline $\mathrm{C}$ & & -1.22 & \\
\hline $\mathrm{H}$ & & -1.06 & 5260392 \\
\hline $\mathrm{H}$ & -0.749458610141 & -1.392123548235 & .769689183 \\
\hline $\mathrm{H}$ & 0.779030960497 & -2.096356787424 & 5.472785498856 \\
\hline $\mathrm{C}$ & & & \\
\hline $\mathrm{H}$ & & & \\
\hline $\mathrm{H}$ & & 1.398915492797 & \\
\hline & -2.514028653004 & 2.100510994040 & 4.921910690660 \\
\hline
\end{tabular}


Cartesian coordinates, number of imaginary frequencies and energy for $\mathbf{6}^{\prime}$

Total energy: -1258.89003

Number of imaginary frequencies: 0

Cartesian coordinates:

\begin{tabular}{|c|c|c|c|}
\hline & 3.667126977569 & -0.048870353169 & -2.3 \\
\hline & 3.685273611244 & -0.051915925073 & \\
\hline & 5.986629276054 & -0.086665060543 & -0.929892164636 \\
\hline$C$ & 6.076313851598 & -0.085011655932 & -2.327491753454 \\
\hline $\mathrm{H}$ & 6.889954348853 & -0.101568554197 & -0.322293866524 \\
\hline H & 7.045282092877 & -0.098452138244 & -2.816628317718 \\
\hline C & 1.473729075289 & -0.020738907821 & 0.486611766037 \\
\hline $\mathrm{C}$ & 2.477797894657 & -0.034760240701 & -0.203513816954 \\
\hline $\mathrm{C}$ & 1.252051392684 & -0.011807844646 & -3.401041957345 \\
\hline $\mathrm{C}$ & 2.424799329469 & -0.029381210326 & \\
\hline $\mathrm{C}$ & -1.301790595041 & & -3.6788 \\
\hline $\mathrm{C}$ & -0.081074550323 & 7959248855 & -3.623280562892 \\
\hline $\mathrm{C}$ & -5.361256627524 & 0.085031234327 & -2.821790212965 \\
\hline $\mathrm{C}$ & 560616089 & 0.08 & 8260 \\
\hline C & -2.6 & 0.0 & 4925 \\
\hline $\mathrm{C}$ & -3.174646087779 & 0.050 & 866934 \\
\hline $\mathrm{H}$ & -6.41 & & \\
\hline $\mathrm{H}$ & & & \\
\hline $\mathrm{C}$ & -1.3 & & 3062 \\
\hline $\mathrm{C}$ & -1.8008 & 0.0 & 8074 \\
\hline $\mathrm{C}$ & -0.932722895066 & & 41271 \\
\hline $\mathrm{C}$ & 0.472836269621 & -0.0077857542 & 77710371 \\
\hline C & 0.9 & -0.0 & \\
\hline $\mathrm{C}$ & 0.06 & -0.0 & 9941 \\
\hline $\mathrm{H}$ & -2.87 & 0.0 & 36291 \\
\hline $\mathrm{H}$ & 1.9995734 & -0.0 & \\
\hline C & & & \\
\hline $\mathrm{C}$ & -2.27 & & -0.9 \\
\hline $\mathrm{O}$ & 0374 & -0.0 & 1589 \\
\hline $\mathrm{O}$ & 52536232 & & 36722 \\
\hline $\mathrm{C}$ & & -1.2176340715 & 5.939470948307 \\
\hline $\mathrm{H}$ & & & \\
\hline $\mathrm{H}$ & -0.73 & & 27728 \\
\hline $\mathrm{H}$ & 0.811861473887 & -2.08 & 5.453285590197 \\
\hline $\mathrm{C}$ & -2.249249875164 & 1.222785326610 & 5.506758413528 \\
\hline $\mathrm{H}$ & & & 6.279918711177 \\
\hline $\mathrm{H}$ & & & \\
\hline $\mathrm{H}$ & -2.53 & 2.08 & 71204107 \\
\hline $\mathrm{N}$ & -4.491937950565 & 0.06995 & -1.812746510794 \\
\hline $\mathrm{C}$ & -3.642197652256 & 0.062539872505 & -4.488176562326 \\
\hline $\mathrm{H}$ & & & \\
\hline $\mathrm{C}$ & & & \\
\hline $\mathrm{H}$ & 4.915071552322 & -0.064037513544 & -4.149383699031 \\
\hline & 4.836966630667 & -0.070835112250 & -0.257480078978 \\
\hline
\end{tabular}


Cartesian coordinates, number of imaginary frequencies and energy for $\mathbf{7}^{\prime}$

Total energy: -1258.88970

Number of imaginary frequencies: 0

Cartesian coordinates:

\begin{tabular}{|c|c|c|c|}
\hline & 3.694168500445 & -0.045698226693 & -2.372879168348 \\
\hline & 3.679555952481 & -0.048316280855 & -0.942696490244 \\
\hline & 4.928620708779 & -0.064511032363 & -0.292578907153 \\
\hline & 6.093660611430 & -0.077396956045 & -1.044945009510 \\
\hline & 5.989378289776 & -0.073789286135 & -2.441706972019 \\
\hline & 4.960710988285 & -0.066394044691 & 0.792618013055 \\
\hline & 7.068191416173 & -0.089877844856 & -0.566660853004 \\
\hline & 6.885117052798 & -0.083710821047 & -3.060285208793 \\
\hline & 1.478994372556 & -0.024210690027 & 0.510586033820 \\
\hline & 2.477619834450 & -0.035221338115 & -0.189024905021 \\
\hline & 1.279129886664 & -0.012431079396 & -3.399574945580 \\
\hline & 2.448931350554 & -0.028592231940 & -3.049825800797 \\
\hline & -1.274557867305 & 0.024555625138 & -3.684004177743 \\
\hline & -0.054430603005 & 0.006529051546 & -3.620841764690 \\
\hline & -5.336986662530 & 0.085320981883 & -2.840443963949 \\
\hline & -4.963775975388 & 0.081634605004 & -4.190352733637 \\
\hline & -2.666825853417 & 0.044962476315 & -3.460966530038 \\
\hline & -3.152976724065 & 0.050051481154 & 421231982 \\
\hline & -6.389522378233 & 0.101277920712 & -2.562403199380 \\
\hline & -5.719045206163 & 0.094517464850 & -4.969908883890 \\
\hline & -1.356800636235 & 0.023968716169 & 3.678472484540 \\
\hline & -1.813183845707 & 77438928 & 852433 \\
\hline & -0.933658367331 & 0.006306273398 & 1.263819080161 \\
\hline & 0.469624236176 & -0.013569627827 & 1.512202486823 \\
\hline & 0.913096554673 & -0.039656565043 & 2.852519610189 \\
\hline & 0.033 & 47077279 & 1893558 \\
\hline & -2.882997467447 & 09685954 & 2.193448494695 \\
\hline & 1.976695140711 & -0.070402896663 & 3.061686830690 \\
\hline & -1.540539320842 & 0.019655448493 & -0.021711324405 \\
\hline & 501782128 & 0.033633198380 & -1.004442376594 \\
\hline & 4.83 & 51756635 & -3.097170190141 \\
\hline & 0.544341618314 & -0.033359006351 & 5.199738189913 \\
\hline & -2.271952336952 & 0.028144647189 & 4.702534135277 \\
\hline & 0.274418709652 & -1.218527932801 & 5.963168710092 \\
\hline & & & 6.939157525061 \\
\hline & -0.801141064330 & -1.380434046991 & 6.081340163424 \\
\hline & 0.728203127543 & -2.094731375337 & 5.483406349403 \\
\hline & -2.297845327587 & 1.230187877216 & 5.486020166779 \\
\hline 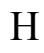 & -3.062052111040 & 1.074106414470 & 6.250141092736 \\
\hline $\mathrm{H}$ & -1.329149762221 & 1.417943254832 & 5.959587060900 \\
\hline H & -2.575423106543 & 2.088622203853 & 4.861818310475 \\
\hline & -4.471125346193 & 0.070243268878 & -1.828379303318 \\
\hline 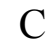 & -3.612301043220 & 0.061309703014 & -4.501176022881 \\
\hline & -3.269434600358 & 0.057807109524 & -5.531163805513 \\
\hline
\end{tabular}


Cartesian coordinates, number of imaginary frequencies and energy for $\mathbf{1 3}^{\mathbf{\prime}}$

Total energy: -1260.061 13

Number of imaginary frequencies: 0

Cartesian coordinates:

\begin{tabular}{|c|c|c|c|}
\hline & -2.660 & 5526585457 & \\
\hline & -2.661241792188 & 0.002299028299 & 3.263490623218 \\
\hline & -1.433357661004 & 0.018234229402 & 3.959511055826 \\
\hline $\mathrm{C}$ & -0.240751760186 & -0.000831962429 & 3.244473480139 \\
\hline$C$ & -0.223978821178 & -0.011780486736 & 1.838223248449 \\
\hline $\mathrm{C}$ & -1.462253429871 & 0.020516294134 & 1.136182364549 \\
\hline $\mathrm{H}$ & -3.613741303940 & 0.025408734180 & 1.355817148904 \\
\hline $\mathrm{H}$ & 0.692629591981 & -0.010615054430 & 3.797027563359 \\
\hline $\mathrm{C}$ & -1.575284393592 & 0.114812123549 & -1.497191357897 \\
\hline C & -1.510301697978 & 0.057950707643 & -0.28422 \\
\hline $\mathrm{C}$ & 2.093432598536 & -0.116720203227 & \\
\hline $\mathrm{C}$ & 1.019352658293 & -0.055004007931 & 1.150072464510 \\
\hline $\mathrm{C}$ & 5.797643101423 & -0.288955002212 & -1.317629703734 \\
\hline $\mathrm{N}$ & 4.77 & -0.9 & -1.87 \\
\hline $\mathrm{C}$ & 3.57 & -0.8 & -1.266 \\
\hline $\mathrm{C}$ & 3.36 & -0.16 & -0.051( \\
\hline $\mathrm{C}$ & 4.4714 & & \\
\hline $\mathrm{C}$ & 5.70 & 0.4 & -0.1 \\
\hline $\mathrm{H}$ & 6.74 & -0.3 & -1.8 \\
\hline $\mathrm{H}$ & 4.34 & 1.0 & 1.441 \\
\hline $\mathrm{H}$ & 6.57 & & \\
\hline $\mathrm{C}$ & & & \\
\hline $\mathrm{C}$ & -2.82 & -0.4 & -4.95 \\
\hline $\mathrm{C}$ & -2.73 & -0.5 & -3.570 \\
\hline $\mathrm{C}$ & -1.683 & 0.155977830041 & -2.9127 \\
\hline $\mathrm{C}$ & -0.746 & 0.862184222896 & -3.7209 \\
\hline $\mathrm{N}$ & -0.8 & & -5.06 \\
\hline $\mathrm{H}$ & $-1.87>y$ & 0.3 & -6.738 \\
\hline $\mathrm{H}$ & -3.62 & -0.94 & -5.487 \\
\hline $\mathrm{H}$ & -3.47 & -1.0 & $-2.982 \varepsilon$ \\
\hline $\mathrm{C}$ & 1.594 & $-2.198063769 c$ & -2.40874 \\
\hline $\mathrm{C}$ & 2.49 & -1.58 & -1.887 \\
\hline $\mathrm{H}$ & 0.792 & -2.72 & -2.874 \\
\hline $\mathrm{C}$ & 1.2553 & 2.18 & -2.615673553562 \\
\hline $\mathrm{C}$ & 0.3416 & 1.572626261358 & -3.120856444573 \\
\hline $\mathrm{H}$ & & & \\
\hline $\mathrm{O}$ & -1.38 & 0.00 & 5.332663191597 \\
\hline $\mathrm{O}$ & -3.86 & 0.02 & 3.924060084005 \\
\hline $\mathrm{C}$ & -1.843693464998 & 1.201445570560 & 5.970597644092 \\
\hline $\mathrm{H}$ & -1.745556395520 & 1.027661522142 & 7.044207620589 \\
\hline $\mathrm{H}$ & & & \\
\hline $\mathrm{H}$ & & & 5.719707225621 \\
\hline $\mathrm{C}$ & -4.178825145234 & -1.171959899372 & 4.653010323438 \\
\hline $\mathrm{H}$ & -5.149671902142 & -0.993850648714 & 5.120133577015 \\
\hline $\mathrm{H}$ & -4253458705863 & & \\
\hline H & & & 5.4209071 \\
\hline
\end{tabular}


Cartesian coordinates, number of imaginary frequencies and energy for $\mathbf{1 6}^{\prime}$

Total energy: -1260.06280

Number of imaginary frequencies: 0

Cartesian coordinates:

\begin{tabular}{|c|c|c|c|}
\hline & $26650^{2}$ & -0.036106446237 & 1.8 \\
\hline & -2.666666996823 & -0.043966865866 & 3.259853732646 \\
\hline & -1.439779226948 & -0.073389411749 & 3.957497037410 \\
\hline & -0.245043796245 & -0.047149703472 & 3.245912921579 \\
\hline & -0.228118180115 & -0.015814111572 & 1.840410115501 \\
\hline & -1.465223556789 & -0.032784418863 & \\
\hline $\mathrm{H}_{\text {- }}$ & -3.616842529502 & -0.033956514949 & 1.348831969558 \\
\hline A & 0.688373833143 & -0.049810280093 & 3.798365088562 \\
\hline $\mathrm{C}$ & -1.569741729567 & -0.094514226484 & -1.497432080797 \\
\hline $\mathrm{C}$ & -1.507462779053 & -0.055424781507 & -0.284573489238 \\
\hline C & 2.087905830437 & & \\
\hline C & 1.014304944206 & 0.041 & 9644 \\
\hline $\mathrm{C}$ & 5.802870385514 & & 985971798 \\
\hline $\mathrm{C}$ & 4.741893569351 & & 963200 \\
\hline $\mathrm{C}$ & 3.47 & & 763 \\
\hline $\mathrm{C}$ & 3.344 & 0.19 & -0.082 \\
\hline $\mathrm{N}$ & & -0.4 & \\
\hline $\mathrm{C}$ & 5.567 & -0.4 & -0.128603041799 \\
\hline $\mathrm{H}$ & & & -1.7 \\
\hline $\mathrm{H}$ & 6.37 & -0.9 & 0.3 \\
\hline $\mathrm{C}$ & & & -5.6 \\
\hline $\mathrm{C}$ & -2.7408 & & 152471 \\
\hline $\mathrm{N}$ & & & \\
\hline $\mathrm{C}$ & -1.6 & -0.1 & 088 \\
\hline $\mathrm{C}$ & -0.66 & -0.8 & 62705 \\
\hline $\mathrm{C}$ & -0.785 & -0.8 & -5.0 \\
\hline $\mathrm{H}$ & & & -6.7 \\
\hline $\mathrm{H}$ & -3.5 & & 2926 \\
\hline $\mathrm{C}$ & 1.4 & 2.2 & -2.34 \\
\hline $\mathrm{C}$ & 2.3 & 1.6 & 06990 \\
\hline $\mathrm{H}$ & 0.6257 & 2.79117 & -2.75 \\
\hline $\mathrm{C}$ & & & 22129968 \\
\hline $\mathrm{C}$ & 0.39 & -1.5 & -3.02 \\
\hline $\mathrm{H}$ & 522 & -2.7 & -2.0 \\
\hline $\mathrm{H}$ & 4.872070960200 & 1.562819983900 & -2.803 \\
\hline $\mathrm{H}$ & -0.058089640991 & -1.424164812936 & -5.657519196685 \\
\hline $\mathrm{O}$ & & & \\
\hline $\mathrm{O}$ & & $-0.0^{\prime} \quad-x-1$ & 103593 \\
\hline $\mathrm{C}$ & & 7875 & 85622 \\
\hline $\mathrm{H}$ & -1.222480764811 & -2.135548765554 & 5.648567368687 \\
\hline $\mathrm{H}$ & & & \\
\hline $\mathrm{H}$ & & & 5.690585205191 \\
\hline $\mathrm{C}$ & & & \\
\hline $\mathrm{H}$ & -5.155252668536 & 0.932780596691 & 5.126072082031 \\
\hline $\mathrm{H}$ & -3.432681541224 & 1.314859154816 & 5.430234485750 \\
\hline & & & \\
\hline
\end{tabular}


Cartesian coordinates, number of imaginary frequencies and energy for $\mathbf{1 9}^{\prime}$

Total energy: -1260.06459

Number of imaginary frequencies: 0

Cartesian coordinates:

\begin{tabular}{|c|c|c|c|}
\hline & -2.593579158996 & -0.155249359674 & 1.972645 \\
\hline & -2.571370213699 & -0.158218553687 & 3.365313517214 \\
\hline & -1.325792889431 & -0.098300644656 & 4.039634069351 \\
\hline 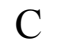 & -0.151246987541 & -0.060802086020 & 3.303317007862 \\
\hline & -0.154318048472 & -0.074742614785 & 1.894993989494 \\
\hline & -1.402687697130 & -0.109723298268 & 1.220443315632 \\
\hline & -3.533431980702 & -0.188478796171 & 1.435826127544 \\
\hline 7 & 0.790395869548 & -0.032820127680 & 3.840613398602 \\
\hline $\mathrm{C}$ & -1.558587182066 & -0.035358148002 & -1.411693649201 \\
\hline $\mathrm{C}$ & -1.477696858788 & -0.083589335495 & -0.199161350933 \\
\hline $\mathrm{C}$ & 2.146458968093 & 175 & 0.60924 \\
\hline$C$ & 1.076707629502 & -0.058536186065 & 1.186055 \\
\hline $\mathrm{C}$ & 5.850787535808 & 0.065618166997 & -1.314747252265 \\
\hline $\mathrm{C}$ & 4.834558576496 & & -1.912337945048 \\
\hline $\mathrm{C}$ & 3.57 & & 3201 \\
\hline $\mathrm{C}$ & 3.401197614658 & -0.036644021920 & -0.069013587284 \\
\hline $\mathrm{N}$ & & & \\
\hline $\mathrm{C}$ & 44958631 & & 5479294454 \\
\hline $\mathrm{H}$ & 597 & & 670 \\
\hline $\mathrm{H}$ & 6.350 & & 0.35 \\
\hline $\mathrm{C}$ & $-1.81 \mathrm{c}$ & & 19305 \\
\hline $\mathrm{C}$ & 1574394 & -0.6620334 & 978331 \\
\hline $\mathrm{C}$ & & & 647208 \\
\hline $\mathrm{C}$ & -1.6 & & -2.8 \\
\hline $\mathrm{C}$ & 7528 & 2720 & 9086223 \\
\hline $\mathrm{N}$ & -0.853 & & -4.970757660074 \\
\hline $\mathrm{H}$ & & & -6.654506788900 \\
\hline $\mathrm{H}$ & -3.5 & & 32817 \\
\hline $\mathrm{H}$ & 0210 & 5263 & 98387 \\
\hline $\mathrm{C}$ & 1.658193473561 & 4325 & 44674 \\
\hline $\mathrm{C}$ & 2.523876251051 & -1.502614825838 & -1.880331940427 \\
\hline $\mathrm{H}$ & & -2.736039134602 & -2.844662968336 \\
\hline $\mathrm{C}$ & 1.13 & & $-2.4 \mathrm{C}$ \\
\hline $\mathrm{C}$ & 4969 & 32226 & 777259 \\
\hline $\mathrm{H}$ & 1.901658989292 & 373470524 & -2.023513873411 \\
\hline $\mathrm{H}$ & 4.994653822352 & -1.192565893861 & -2.849563907838 \\
\hline $\mathrm{O}$ & & & \\
\hline $\mathrm{O}$ & -1.2453( & -0.15278 & \\
\hline $\mathrm{C}$ & -4.949722907046 & -0.319934297679 & 3.530012616304 \\
\hline $\mathrm{H}$ & -5.676207294592 & -0.379065387608 & 4.341973665461 \\
\hline $\mathrm{H}$ & -5.163180990135 & & 2.912851924716 \\
\hline $\mathrm{H}$ & -5.021289387677 & -1.222674154392 & 2.910368283931 \\
\hline $\mathrm{C}$ & & & 6.114164853793 \\
\hline $\mathrm{H}$ & -1.553540484030 & 0.787755890246 & 7.170597040377 \\
\hline $\mathrm{H}$ & -1.214180441454 & 1.896125334486 & 5.811252661007 \\
\hline & & 1.11605172 & 5.95506405 \\
\hline
\end{tabular}


Cartesian coordinates, number of imaginary frequencies and energy for $\mathbf{2 1}$

Total energy: -997.785815224

Number of imaginary frequencies: 0

Cartesian coordinates:

$\begin{array}{lrrr}\mathrm{C} & 3.488277647637 & -0.005577573823 & -1.368383535658 \\ \mathrm{C} & 3.501480267796 & -0.005775935741 & 0.061822576031 \\ \mathrm{C} & 4.745936726496 & -0.007283296901 & 0.722763475704 \\ \mathrm{C} & 5.937993886601 & -0.008483188970 & 0.007758353614 \\ \mathrm{C} & 5.919627713240 & -0.008250341723 & -1.392368701871 \\ \mathrm{H} & 4.756903133738 & -0.007471499551 & 1.808230590546 \\ \mathrm{H} & 6.884719350093 & -0.009615541327 & 0.540473831705 \\ \mathrm{H} & 6.851384797603 & -0.009191655952 & -1.950674759765 \\ \mathrm{C} & 1.306090171375 & -0.003324492430 & 1.528096624076 \\ \mathrm{C} & 2.301488885925 & -0.004474511098 & 0.823727735449 \\ \mathrm{C} & 1.066025262938 & -0.002763823381 & -2.366860738896 \\ \mathrm{C} & 2.240462540034 & -0.004173709393 & -2.029712709828 \\ \mathrm{C} & -1.489811048961 & 0.001231822681 & -2.630574714372 \\ \mathrm{C} & -0.268910419624 & -0.000882305239 & -2.581884006671 \\ \mathrm{C} & -5.640096980955 & 0.007517736851 & -1.857183895423 \\ \mathrm{C} & -5.183020045839 & 0.005551555610 & -3.180728348553 \\ \mathrm{C} & -2.882172387522 & 0.003270118812 & -2.394509211607 \\ \mathrm{C} & -3.343791701505 & 0.005262770099 & -1.040785735612 \\ \mathrm{C} & -4.732842292935 & 0.007394343600 & -0.804034854293 \\ \mathrm{H} & -6.706212228863 & 0.009164846594 & -1.648685135761 \\ \mathrm{H} & -5.892343360422 & 0.005656028784 & -4.003369358667 \\ \mathrm{H} & -5.084090446728 & 0.008929615457 & 0.223088069189 \\ \mathrm{C} & -1.472105146772 & 0.004745615634 & 4.738467652592 \\ \mathrm{C} & -1.958861617023 & 0.006039621098 & 3.439830754397 \\ \mathrm{C} & -1.095979354867 & 0.003484691347 & 2.319539588605 \\ \mathrm{C} & 0.312205654327 & -0.000458320048 & 2.546364619637 \\ \mathrm{C} & 0.779670949443 & -0.001589897730 & 3.880942985656 \\ \mathrm{C} & -0.090241055573 & 0.001077527246 & 4.961052843281 \\ \mathrm{H} & -3.028613327413 & 0.009035080609 & 3.257199827407 \\ \mathrm{H} & 1.852662994488 & -0.004596232787 & 4.043456906305 \\ \mathrm{C} & -1.719856847235 & 0.005028570566 & 1.040689043173 \\ \mathrm{C} & -2.443731310771 & 0.005099265780 & 0.059383254259 \\ \mathrm{C} & -3.819028372740 & 0.003479162082 & -3.444640783344 \\ \mathrm{C} & 4.707491509619 & -0.006830655021 & -2.071228962270 \\ \mathrm{H} & -3.454420446578 & 0.001971341541 & -4.467046727344 \\ \mathrm{H} & 4.682360047154 & -0.006662561844 & -3.156412601138 \\ & -2.164600701755 & 0.006674284955 & 5.575388600725 \\ \mathrm{H} & 0.304434390032 & 0.000217794087 & 5.973090908343\end{array}$


Cartesian coordinates, number of imaginary frequencies and energy for $\mathbf{2 2}$

Total energy: -1029.85273955

Number of imaginary frequencies: 0

Cartesian coordinates:

$\begin{array}{lrrr}\mathrm{C} & 3.491540000283 & -0.005758339677 & -1.369276364379 \\ \mathrm{C} & 3.490788763000 & -0.006099931706 & 0.060748469478 \\ \mathrm{C} & 4.745606741984 & -0.007679502015 & 0.699695507689 \\ \mathrm{C} & 5.903640361821 & -0.008696849541 & -0.063466061200 \\ \mathrm{C} & 5.786099862926 & -0.008146712954 & -1.459275178645 \\ \mathrm{H} & 4.787797719589 & -0.008050508584 & 1.784522086364 \\ \mathrm{H} & 6.882714580123 & -0.009903064128 & 0.405574965652 \\ \mathrm{H} & 6.676044695583 & -0.008916530976 & -2.086181533785 \\ \mathrm{C} & 1.303094802298 & -0.003665404798 & 1.534087161719 \\ \mathrm{C} & 2.295487051180 & -0.004881904928 & 0.825637331599 \\ \mathrm{C} & 1.066910160501 & -0.002786953667 & -2.372140064581 \\ \mathrm{C} & 2.240203880523 & -0.004263564768 & -2.035116284124 \\ \mathrm{C} & -1.487869383233 & 0.001351493553 & -2.635622357064 \\ \mathrm{C} & -0.268093457423 & -0.000831453227 & -2.587174359296 \\ \mathrm{C} & -5.585117293813 & 0.007617037196 & -1.914017713999 \\ \mathrm{C} & -5.035237835887 & 0.005369874287 & -3.202321542936 \\ \mathrm{C} & -2.884990067546 & 0.003470931087 & -2.396380138960 \\ \mathrm{C} & -3.333304363151 & 0.005593858430 & -1.038447458211 \\ \mathrm{C} & -4.725286389091 & 0.007741161220 & -0.825831539905 \\ \mathrm{H} & -6.661951427547 & 0.009234311722 & -1.776130455010 \\ \mathrm{H} & -5.683323289078 & 0.005221347640 & -4.076963883507 \\ \mathrm{H} & -5.105978606719 & 0.009444693361 & 0.190878562860 \\ \mathrm{C} & -1.473096998265 & 0.004936134782 & 4.744877558272 \\ \mathrm{C} & -1.960429817290 & 0.006424624381 & 3.446370619521 \\ \mathrm{C} & -1.097010788723 & 0.003684105782 & 2.326809905519 \\ \mathrm{C} & 0.310902446287 & -0.000648532090 & 2.553590504828 \\ \mathrm{C} & 0.779108238879 & -0.001973690884 & 3.887643547462 \\ \mathrm{C} & -0.091308840354 & 0.000881132748 & 4.967449868500 \\ \mathrm{H} & -3.030164458953 & 0.009758474655 & 3.264108164185 \\ \mathrm{H} & 1.851968522344 & -0.005317932761 & 4.050500978892 \\ \mathrm{C} & -1.718895174765 & 0.005383732037 & 1.047318379413 \\ \mathrm{C} & -2.438633290758 & 0.005515336416 & 0.063081697792 \\ \mathrm{H} & -2.165319776993 & 0.007055996175 & 5.581850525653 \\ \mathrm{H} & 0.303092512663 & -0.000174998931 & 5.979451383556 \\ \mathrm{~N} & -3.727074746428 & 0.003381634763 & -3.448586735440 \\ \mathrm{~N} & 4.621427299670 & -0.006748960212 & -2.103850368246\end{array}$


Cartesian coordinates, number of imaginary frequencies and energy for $\mathbf{2 3}$

Total energy: -1226.82188269

Number of imaginary frequencies: 0

Cartesian coordinates:

\begin{tabular}{|c|c|c|c|}
\hline & 3.656137667095 & -0.005407463061 & -2.407547674134 \\
\hline $\mathrm{C}$ & 3.670224085496 & -0.005023699421 & -0.977322998415 \\
\hline $\mathrm{C}$ & 4.915344211723 & -0.005279161874 & -0.317315813917 \\
\hline $\mathrm{C}$ & 6.106761969719 & -0.006245160709 & -1.033220473978 \\
\hline $\mathrm{C}$ & 6.087574462110 & -0.006924649886 & -2.433421662150 \\
\hline $\mathrm{H}$ & 4.927068696412 & -0.004382518215 & 0.768166552354 \\
\hline $\mathrm{H}$ & 7.053876329628 & -0.006324053393 & -0.501172185629 \\
\hline $\mathrm{H}$ & 7.019038814359 & -0.007687677220 & -2.992212588286 \\
\hline $\mathrm{C}$ & 1.476106050375 & -0.003138110842 & 0.490461777613 \\
\hline $\mathrm{C}$ & 2.470982058045 & -0.004214504106 & -0.214671089163 \\
\hline $\mathrm{C}$ & 1.233276469148 & -0.003295597570 & -3.404946230012 \\
\hline $\mathrm{C}$ & 2.407777593494 & -0.004483312098 & -3.067953612363 \\
\hline $\mathrm{C}$ & -1.322648733511 & 0.000210357589 & -3.668837848420 \\
\hline $\mathrm{C}$ & -0.101735924377 & -0.001627533693 & -3.619983913605 \\
\hline $\mathrm{C}$ & -5.473456689517 & 0.003901593098 & -2.898509219994 \\
\hline $\mathrm{C}$ & -5.015573504736 & 0.002826666113 & -4.221866235933 \\
\hline $\mathrm{C}$ & -2.715238103123 & 0.001751904463 & -3.433822950378 \\
\hline $\mathrm{C}$ & -3.177705517601 & 0.003181927129 & -2.080359735895 \\
\hline $\mathrm{C}$ & -4.567093783466 & 0.004046553922 & -1.844705183114 \\
\hline $\mathrm{H}$ & -6.539732382745 & 0.004483298654 & -2.690767096177 \\
\hline $\mathrm{H}$ & -5.724464850761 & 0.002725751492 & -5.044877028280 \\
\hline $\mathrm{H}$ & -4.919069058788 & 0.004511410889 & -0.817805294821 \\
\hline $\mathrm{C}$ & -1.311308655627 & 0.022726602436 & 3.702003001462 \\
\hline $\mathrm{C}$ & -1.787599841902 & 0.021862940978 & 2.399494691770 \\
\hline $\mathrm{C}$ & -0.928030863972 & 0.002868553585 & 1.278582644875 \\
\hline $\mathrm{C}$ & 0.479614412427 & -0.001044738536 & 1.505322100416 \\
\hline $\mathrm{C}$ & 0.943732832468 & -0.018543212865 & 2.839470269047 \\
\hline $\mathrm{C}$ & 0.082538179510 & -0.017952248012 & 3.926542060790 \\
\hline $\mathrm{H}$ & -2.860900208509 & 0.040168346089 & 2.245479172080 \\
\hline $\mathrm{H}$ & 2.011105365549 & -0.036828226544 & 3.030287322616 \\
\hline $\mathrm{C}$ & -1.555451401341 & 0.003565733003 & 0.002148350289 \\
\hline $\mathrm{C}$ & -2.278592939635 & 0.003541681134 & -0.979716180491 \\
\hline $\mathrm{O}$ & 0.612120647079 & -0.011590604405 & 5.195000311026 \\
\hline $\mathrm{O}$ & -2.212410556713 & 0.017762484231 & 4.740017111260 \\
\hline $\mathrm{C}$ & 0.381471471580 & -1.208229852825 & 5.951861840329 \\
\hline $\mathrm{H}$ & 0.849463961262 & -1.043801286637 & 6.924783969822 \\
\hline $\mathrm{H}$ & -0.688577966234 & -1.398602147708 & 6.079793439921 \\
\hline $\mathrm{H}$ & 0.852204341829 & -2.069527032080 & 5.461292099979 \\
\hline $\mathrm{C}$ & -2.230853212657 & 1.215329435403 & 5.529622733623 \\
\hline $\mathrm{H}$ & -2.980768006635 & 1.051984928956 & 6.306525200416 \\
\hline $\mathrm{H}$ & -1.255081277046 & 1.406007147408 & 5.986924326905 \\
\hline $\mathrm{H}$ & -2.523491924013 & 2.075978622000 & 4.915028438959 \\
\hline $\mathrm{C}$ & -3.651337727178 & 0.001718571365 & -4.484690112996 \\
\hline $\mathrm{C}$ & 4.874866035727 & -0.006454922662 & -3.111326505822 \\
\hline $\mathrm{H}$ & -3.285925806722 & 0.000688310607 & -5.506829186126 \\
\hline $\mathrm{H}$ & 4.848889647835 & -0.006780025250 & -4.196508698628 \\
\hline
\end{tabular}


Cartesian coordinates, number of imaginary frequencies and energy for $\mathbf{5}^{\prime} \cdot \mathbf{H}$

Total energy: -1259.28091319

Number of imaginary frequencies: 0

Cartesian coordinates:

\begin{tabular}{|c|c|c|c|}
\hline & 3.710675916443 & -0.022270754089 & -2.319010360727 \\
\hline $\mathrm{C}$ & 3.699613575209 & -0.018116646536 & -0.890777454567 \\
\hline $\mathrm{C}$ & 4.957431934533 & -0.019202186853 & -0.251099864211 \\
\hline $\mathrm{C}$ & 6.109937124311 & -0.024609526118 & -1.018796853032 \\
\hline $\mathrm{C}$ & 5.997523165119 & -0.028745283697 & -2.420159993990 \\
\hline $\mathrm{H}$ & 5.003794662951 & -0.015611279884 & 0.832961582768 \\
\hline $\mathrm{H}$ & 7.089500901457 & -0.025545562104 & -0.551620467718 \\
\hline $\mathrm{H}$ & 6.888274548575 & -0.033054395022 & -3.044022860581 \\
\hline $\mathrm{C}$ & 1.492903650152 & -0.008024262915 & 0.550093542672 \\
\hline $\mathrm{C}$ & 2.500593871308 & -0.013045675175 & -0.137470484392 \\
\hline $\mathrm{C}$ & 1.307707452478 & -0.015404680993 & -3.385477319346 \\
\hline $\mathrm{C}$ & 2.464544689141 & -0.019301009977 & -2.985856957276 \\
\hline $\mathrm{C}$ & -1.235996007044 & -0.003397762142 & -3.734821989940 \\
\hline $\mathrm{C}$ & -0.014493591814 & -0.009475511857 & -3.609574592842 \\
\hline $\mathrm{C}$ & -5.364265267090 & 0.022614177249 & -3.001872236325 \\
\hline $\mathrm{C}$ & -4.851034658783 & 7800665 & -4.286060572539 \\
\hline $\mathrm{C}$ & -2.594154896277 & 0.005393691774 & -3.442062780968 \\
\hline $\mathrm{C}$ & -3.088450161264 & 0.016169070965 & -2.095972746512 \\
\hline $\mathrm{C}$ & -4.485806233964 & 0.024173368963 & -1.917149480195 \\
\hline $\mathrm{H}$ & -6.437352372012 & 0.028832275182 & -2.852715820421 \\
\hline $\mathrm{H}$ & -5.456372376636 & 0.010406128811 & -5.18391 \\
\hline $\mathrm{H}$ & -4.876034507150 & 0.031315166648 & -0.904889781458 \\
\hline $\mathrm{C}$ & -1.360098595430 & 0.034776732329 & 3.710977220315 \\
\hline $\mathrm{C}$ & -1.808166728803 & 0.037936347246 & 2.397786379443 \\
\hline $\mathrm{C}$ & -0.923066046270 & 0.011727775142 & 1.2969100 \\
\hline $\mathrm{C}$ & 0.482731272530 & -0.000529785862 & 1.547823366516 \\
\hline $\mathrm{C}$ & 0.918369890369 & -0.028269244407 & 2.887473631699 \\
\hline $\mathrm{C}$ & 0.034148656953 & -0.031881014616 & 3.9613 \\
\hline $\mathrm{H}$ & -2.878287204514 & 0.071652557390 & 2.2263 \\
\hline $\mathrm{H}$ & 1.980051277998 & -0.060898598392 & 3.104149095963 \\
\hline $\mathrm{C}$ & -1.523473192397 & 0.015441325729 & 0.014563904519 \\
\hline $\mathrm{C}$ & -2.213639630446 & 0.016751428688 & -0.993674684567 \\
\hline $\mathrm{N}$ & 4.834812823593 & -0.027502090829 & -3.062404545081 \\
\hline $\mathrm{N}$ & -3.511923620069 & 0.004268318973 & -4.459390214845 \\
\hline $\mathrm{O}$ & 0.552370211893 & -0.046087775641 & 5.219555557942 \\
\hline $\mathrm{O}$ & -2.284226545159 & 0.049472020263 & 4.714932365828 \\
\hline $\mathrm{C}$ & 0.210630437436 & -1.179387649891 & 6.044339777091 \\
\hline $\mathrm{H}$ & 0.697636932817 & -1.000072855486 & 7.003383594834 \\
\hline $\mathrm{H}$ & -0.870400110806 & -1.265298430964 & 6.178829612439 \\
\hline $\mathrm{H}$ & 0.604563475644 & -2.098947798626 & 5.597250717434 \\
\hline $\mathrm{C}$ & -2.278277495917 & 1.220577287992 & 5.555829586216 \\
\hline $\mathrm{H}$ & -3.056608052468 & 1.052168790304 & 6.301098720741 \\
\hline $\mathrm{H}$ & -1.310428511086 & 1.355036778445 & 6.046404947504 \\
\hline $\mathrm{H}$ & -2.520717047345 & 2.110739824240 & 4.964089270367 \\
\hline $\mathrm{H}$ & -3.138330158876 & -0.003428224197 & -5.404659675776 \\
\hline
\end{tabular}


Cartesian coordinates, number of imaginary frequencies and energy for $\mathbf{6}^{\prime} \cdot \mathbf{H}$

Total energy: -1259.28191610

Number of imaginary frequencies: 0

Cartesian coordinates:

\begin{tabular}{|c|c|c|c|}
\hline & 3.716821413290 & 0.020088591237 & -2.347345134720 \\
\hline 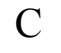 & 3.703548684722 & 0.009607593049 & -0.917974174177 \\
\hline$C$ & 5.999914630677 & 0.011335541174 & -0.837586826994 \\
\hline$C$ & 6.119579868109 & 0.022203301561 & -2.235275733611 \\
\hline $\mathrm{H}$ & 6.889412704364 & 0.007471280414 & \\
\hline $\mathrm{H}$ & 7.099436823209 & 0.026981101352 & -2.701003649288 \\
\hline$C$ & 1.466495742655 & -0.000378889398 & 0.490249715508 \\
\hline $\mathrm{C}$ & 2.480568227660 & 0.003784983538 & -0.185099046078 \\
\hline $\mathrm{C}$ & 1.327691107887 & 0.021923304807 & -3.422007365262 \\
\hline $\mathrm{C}$ & 2.485335794383 & 0.022557610540 & -3.027353960279 \\
\hline $\mathrm{C}$ & -1.221738584352 & 0.013010309290 & -3.730803959744 \\
\hline $\mathrm{C}$ & -0.001631531452 & 0.018032904684 & -3.641459256954 \\
\hline $\mathrm{C}$ & -5.343529334420 & -0.020604805202 & -2.923520078047 \\
\hline $\mathrm{C}$ & 964168 & -0.01164 & 42040014766 \\
\hline $\mathrm{C}$ & 892678 & 0.002 & -3.49972 \\
\hline $\mathrm{C}$ & 54928303 & -0.0055 & -2.138129 \\
\hline $\mathrm{H}$ & -6.3801 & -0.0304 & 465470 \\
\hline $\mathrm{H}$ & -5.670114463516 & -0.014009116660 & \\
\hline $\mathrm{C}$ & -1.34 & 0.00 & 417 \\
\hline $\mathrm{C}$ & -1.814 & 0.012 & 2.38738 \\
\hline $\mathrm{C}$ & -0.94 & -0.004 & 1.272283601150 \\
\hline $\mathrm{C}$ & 0.470426985657 & -0.004548799420 & \\
\hline $\mathrm{C}$ & 0.92 & -0.03 & 3463 \\
\hline $\mathrm{C}$ & 8895 & -0.05 & 19202 \\
\hline $\mathrm{H}$ & -2.88 & 0.04 & 2.232957006781 \\
\hline $\mathrm{H}$ & 4793 & -0.0581 & 3.030966563764 \\
\hline $\mathrm{C}$ & -1.55 & -0.0 & \\
\hline $\mathrm{C}$ & -2.2 & -0.0 & 0600094 \\
\hline $\mathrm{O}$ & 0723 & -0.07 & 88696 \\
\hline $\mathrm{O}$ & -2.256 & 0.02 & 4.710359125882 \\
\hline $\mathrm{C}$ & 0.23825105 & -1.1822 & 6.019773662650 \\
\hline $\mathrm{H}$ & & -0.98 & 82365 \\
\hline $\mathrm{H}$ & -0.84 & -1.2 & 6.166306883223 \\
\hline $\mathrm{H}$ & 0.613 & -2.116 & 5.589134656446 \\
\hline $\mathrm{C}$ & -2.242662559715 & 1.199235864167 & 5.542328032714 \\
\hline $\mathrm{H}$ & & 1.029245363470 & 6.306510059269 \\
\hline $\mathrm{H}$ & & & \\
\hline $\mathrm{H}$ & -2.50630 & 2.0824 & 4.949309437769 \\
\hline $\mathrm{C}$ & 4.963935172068 & 0.026497479457 & -2.999595668755 \\
\hline $\mathrm{H}$ & 5.002503535196 & 0.034568148249 & -4.084037769398 \\
\hline $\mathrm{N}$ & & & \\
\hline $\mathrm{C}$ & -3.564021450127 & -0.000008738236 & -4.52510386574 \\
\hline $\mathrm{H}$ & -3.220502160289 & 0.006767730056 & -5.554228820651 \\
\hline $\mathrm{N}$ & -4.417245213331 & -0.017364398865 & -1.937841172338 \\
\hline $\mathrm{H}$ & -4.731912317227 & -0.025284320727 & -0.972176836702 \\
\hline
\end{tabular}


Cartesian coordinates, number of imaginary frequencies and energy for $\mathbf{7}^{\prime} \cdot \mathbf{H}$

Total energy: -1259.28175232

Number of imaginary frequencies: 0

Cartesian coordinates:

\begin{tabular}{|c|c|c|c|}
\hline & 3.699335094899 & 0.013679357422 & -2.324872175159 \\
\hline & 3.696350216828 & 0.008589212702 & -0.892316094151 \\
\hline & 5.995085287399 & 0.009461849816 & -0.841809257858 \\
\hline & 6.102446523195 & 0.014985086737 & -2.242007303536 \\
\hline $\mathrm{H}$ & 6.891470109090 & 0.007541324787 & \\
\hline & 7.077850587973 & 0.017496099621 & -2.716647073611 \\
\hline 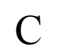 & 1.470935957628 & 0.004179059617 & 0.528349421425 \\
\hline$C$ & 2.482343543183 & 0.005802352686 & -0.151738911162 \\
\hline $\mathrm{C}$ & 1.310874180898 & 0.012765859894 & 401674899638 \\
\hline $\mathrm{C}$ & 2.466017558650 & 0.014135040465 & -2.990806489874 \\
\hline$C$ & -1.234526867701 & 0.004074963807 & -3.738922324824 \\
\hline$C$ & -0.011861760123 & 0.008634279128 & -3.619177593308 \\
\hline $\mathrm{C}$ & -5.366571100466 & -0.025774902450 & -3.022984526741 \\
\hline $\mathrm{C}$ & -4.847584052000 & 2708863 & 6215213 \\
\hline $\mathrm{C}$ & -2.593517728759 & -0.004880162047 & \\
\hline $\mathrm{C}$ & -3.094342879322 & -0.009803352528 & -2.107122839516 \\
\hline $\mathrm{C}$ & -4.492476313835 & -0.020751158567 & \\
\hline $\mathrm{H}$ & -6.4402 & 0595 & 259698 \\
\hline $\mathrm{H}$ & -5.448914215370 & 3265857517 & 05216675022 \\
\hline $\mathrm{H}$ & -4.886585588656 & -0.025587618145 & \\
\hline $\mathrm{C}$ & -1.355075218174 & 0.021484849122 & 3.709227922340 \\
\hline $\mathrm{C}$ & -1.8154 & 4827 & \\
\hline $\mathrm{C}$ & -0.9385 & 0.0 & 170 \\
\hline $\mathrm{C}$ & 0.468836147235 & 0.002622382053 & 11079 \\
\hline $\mathrm{C}$ & 0.918518892289 & -0.018791992240 & 2.868083530613 \\
\hline $\mathrm{C}$ & 0.042012390481 & -0.031601887204 & \\
\hline $\mathrm{H}$ & -2.887309053839 & 2277 & 65988 \\
\hline $\mathrm{H}$ & 1.983196251649 & 61225179 & 6277710 \\
\hline $\mathrm{C}$ & -1.542731953167 & -0.001 & 0.012642721016 \\
\hline $\mathrm{C}$ & -2.225417005079 & -0.004391612029 & -1.000635608375 \\
\hline $\mathrm{N}$ & -3.507 & & \\
\hline $\mathrm{O}$ & 0.56825 & -0.04 & 387972 \\
\hline $\mathrm{O}$ & -2.271360195650 & 0.028584077698 & 4.719936086789 \\
\hline $\mathrm{C}$ & 0.254136921127 & -1.186231657854 & 6.023140197083 \\
\hline $\mathrm{H}$ & 0.740637552177 & -1.001406478883 & 6.981390007562 \\
\hline $\mathrm{H}$ & -0.824 & & \\
\hline $\mathrm{H}$ & 0.666381640891 & -2.094632168978 & 5.569923492300 \\
\hline $\mathrm{C}$ & -2.259165174043 & 1.190712460309 & 5.573363823248 \\
\hline $\mathrm{H}$ & -3.029626615125 & 1.012195923191 & 6.324426817798 \\
\hline $\mathrm{H}$ & & 1.322724890047 & 6.055286120887 \\
\hline $\mathrm{H}$ & -2.510035529356 & 2.086217555055 & 4.993316768551 \\
\hline $\mathrm{C}$ & 4.940262629820 & 0.016995994936 & -2.993079745077 \\
\hline $\mathrm{H}$ & 4.966342285960 & 0.020958686282 & -4.077927807685 \\
\hline $\mathrm{N}$ & 4.839504105969 & 0.006382604844 & -0.180043523858 \\
\hline $\mathrm{H}$ & -3.130556092420 & -0.006420154663 & -5.416066664075 \\
\hline
\end{tabular}


Cartesian coordinates, number of imaginary frequencies and energy for $\mathbf{7}^{\prime} \cdot \mathbf{H}^{\prime}$

Total energy: -1259.28067635

Number of imaginary frequencies: 0

Cartesian coordinates:

\begin{tabular}{|c|c|c|c|}
\hline$C$ & 3.650707397201 & -0.000520394204 & -2.376977020709 \\
\hline $\mathrm{C}$ & 3.599980459494 & 0.007081238122 & -0.942929843397 \\
\hline $\mathrm{C}$ & 6.014215341886 & 0.024264516889 & -0.879530749003 \\
\hline $\mathrm{C}$ & 6.090728033952 & 0.016001718148 & -2.258511504435 \\
\hline $\mathrm{H}$ & 6.875822040261 & 0.034662365133 & -0.223505402245 \\
\hline $\mathrm{H}$ & 7.059323815512 & 0.019491099627 & -2.743699979146 \\
\hline $\mathrm{C}$ & 1.436653664139 & 0.003023445987 & 0.533470093144 \\
\hline $\mathrm{C}$ & 2.431422226313 & 0.003048490160 & -0.182563527135 \\
\hline $\mathrm{C}$ & 1.257987477314 & -0.017387533429 & -3.407815167778 \\
\hline $\mathrm{C}$ & 2.434835505094 & -0.011093959272 & -3.075383136685 \\
\hline $\mathrm{C}$ & -1.287373595196 & -0.025323446099 & -3.692113448617 \\
\hline $\mathrm{C}$ & -0.066595243966 & -0.022809765976 & -3.656136933911 \\
\hline $\mathrm{C}$ & -5.390769118305 & -0.027642358081 & -3.065272985463 \\
\hline $\mathrm{C}$ & -4.812642557509 & -0.037368781579 & -4.344131110513 \\
\hline $\mathrm{C}$ & -2.688983907167 & -0.026053169210 & -3.484787869602 \\
\hline $\mathrm{C}$ & -3.163042038553 & -0.015259643269 & -2.137882774727 \\
\hline $\mathrm{C}$ & -4.560817127580 & -0.016568409065 & -1.955529012602 \\
\hline $\mathrm{H}$ & -6.470210433577 & -0.029015536360 & -2.953768550200 \\
\hline $\mathrm{H}$ & -5.440210252253 & -0.046127921066 & -5.232368306422 \\
\hline $\mathrm{H}$ & -4.968103841450 & -0.009274001547 & -0.949674670503 \\
\hline $\mathrm{C}$ & -1.399235189443 & 0.064432723318 & 3.675808759406 \\
\hline $\mathrm{C}$ & -1.851388446238 & 0.044938314157 & 2.358657324622 \\
\hline $\mathrm{C}$ & -0.978237163134 & 0.010419570124 & 1.255412252247 \\
\hline $\mathrm{C}$ & 0.428697743430 & 0.005828964195 & 1.517355551758 \\
\hline $\mathrm{C}$ & 0.873497473231 & -0.012563605102 & 2.862253163984 \\
\hline $\mathrm{C}$ & -0.003727392795 & -0.004024277595 & 3.933450901176 \\
\hline $\mathrm{H}$ & -2.921874527669 & 0.074490544611 & 2.191093005625 \\
\hline $\mathrm{H}$ & 1.935740891204 & -0.046406060805 & 3.077689463705 \\
\hline $\mathrm{C}$ & -1.575505898454 & 0.003453670664 & -0.031898606309 \\
\hline $\mathrm{C}$ & -2.288169706987 & -0.004326029769 & -1.021851303092 \\
\hline $\mathrm{N}$ & -3.499548733032 & -0.036761577554 & -4.558747008811 \\
\hline $\mathrm{O}$ & 0.506840282753 & -0.021722504698 & 5.199415398432 \\
\hline $\mathrm{O}$ & -2.329134325482 & 0.089189523752 & 4.662938412125 \\
\hline $\mathrm{C}$ & 0.218336600817 & -1.206281464096 & 5.970103549889 \\
\hline $\mathrm{H}$ & 0.675684734684 & -1.041822659714 & 6.946412478791 \\
\hline $\mathrm{H}$ & -0.859071521460 & -1.357730480990 & 6.080691818718 \\
\hline $\mathrm{H}$ & 0.668583513621 & -2.084894002394 & 5.494189367515 \\
\hline $\mathrm{C}$ & -2.271638885977 & 1.187738375279 & 5.599076214896 \\
\hline $\mathrm{H}$ & -3.064364140761 & 0.994075029200 & 6.321953329223 \\
\hline $\mathrm{H}$ & -1.302724048158 & 1.237702374369 & 6.100247036428 \\
\hline $\mathrm{H}$ & -2.471102611711 & 2.129227113250 & 5.075940010330 \\
\hline $\mathrm{C}$ & 4.907734834662 & 0.003818717685 & -3.002592244781 \\
\hline $\mathrm{H}$ & 4.945407353327 & -0.002272380628 & -4.086909065407 \\
\hline $\mathrm{N}$ & 4.801845617888 & 0.019628635569 & -0.279838384488 \\
\hline $\mathrm{H}$ & 4.759350258882 & 0.027127510885 & 0.734893298036 \\
\hline
\end{tabular}


Cartesian coordinates, number of imaginary frequencies and energy for $\mathbf{5}^{\mathbf{2}} \mathbf{2} \mathbf{H}$

Total energy: -1259.58182786

Number of imaginary frequencies: 0

Cartesian coordinates:

\begin{tabular}{|c|c|c|c|}
\hline & 3.613897038198 & 0.006264965839 & -2.3673838004688 \\
\hline & 3.635583473007 & 0.003553425401 & -0.940717076797 \\
\hline & 4.906065220821 & 0.008459451553 & -0.312595835053 \\
\hline & 6.081241766419 & 0.014888023095 & -1.053372160530 \\
\hline & 6.011748649294 & 0.017010246974 & -2.442489764560 \\
\hline & 4.946829603767 & 0.007490119960 & 0.771610580197 \\
\hline & 7.050327389251 & 0.018654804525 & -0.567927888913 \\
\hline & 6.878944015418 & 0.022094399045 & -3.092014680380 \\
\hline & 1.465990414502 & -0.004803483804 & 0.540153902120 \\
\hline & 2.451053409824 & -0.002918845452 & -0.184312454916 \\
\hline & 1.228033747585 & -0.000654320975 & -3.395312261883 \\
\hline & 2.409745812651 & 0.002757696401 & -3.076060138749 \\
\hline & -1.321971637497 & -0.007061405994 & -3.677140957366 \\
\hline & -0.099780474479 & -0.004225124867 & -3.609187432057 \\
\hline & -5.442904522722 & -0.017201814897 & -2.909608393515 \\
\hline & -4.940738248699 & -0.021047910251 & -4.206641897954 \\
\hline & -2.687746915591 & -0.009868752840 & 2412670996 \\
\hline & -3.156314926458 & -0.005354318336 & 615471 \\
\hline & -4.559771648273 & -0.009671744064 & -1.837297611444 \\
\hline & -6.515407768942 & -0.020506061837 & -2.753009707444 \\
\hline & -5.560120094482 & -0.027073711870 & -5.095611010570 \\
\hline & -4.938919195909 & -0.007352852425 & 857319 \\
\hline & -1.323422164543 & 0.050073133330 & 3.749102871644 \\
\hline & -1.796882352886 & 0.040738319769 & 2.438643927157 \\
\hline & -0.938936762666 & 0.006288153463 & 1.322102418605 \\
\hline & 0.476300234304 & -0.00 & 68756418 \\
\hline & 0.940248102769 & -0.037405445959 & 2.879575864110 \\
\hline & 0.079244270305 & -0.045262138478 & 96457949 \\
\hline & -2.870360388700 & 0.083811590369 & 2.293551499524 \\
\hline & 2.004982837779 & -0.080444254124 & 3.078949446054 \\
\hline & -1.56145 & 0.00 & 0.05250 \\
\hline & -2.269208943763 & 0.002247937180 & -0.944633123111 \\
\hline & 4.807277096510 & 0.012827354859 & -3.039770659187 \\
\hline & -3.609637288193 & -0.017433534093 & -4.395508932984 \\
\hline & 0.630446849654 & -0.090035081973 & 5.207702536038 \\
\hline & -2.233779230277 & 0.096219863108 & 4.746232692743 \\
\hline & 0.249680634152 & -1.188995958159 & 6.073727733700 \\
\hline & 0.745977274203 & -0.991701442860 & 7.023312432984 \\
\hline & -0.832437784732 & & 6.211176708089 \\
\hline & 0.615537488949 & -2.129774451061 & 5.650468860261 \\
\hline & -2.143991735187 & 1.196294897334 & 5.686695609230 \\
\hline & -2.913403119602 & 1.000051684361 & 6.432645549566 \\
\hline & -1.159757478489 & 1.243442504739 & 6.156930819041 \\
\hline & -2.358247720919 & 2.136494418527 & 5.168832011734 \\
\hline & 4.767338011390 & 0.014710828201 & -4.056832212276 \\
\hline & & -0.020589616 & -5.3485849 \\
\hline
\end{tabular}


Cartesian coordinates, number of imaginary frequencies and energy for $\mathbf{6}^{\prime} \cdot \mathbf{2} \mathbf{H}$

Total energy: -1259.58776975

Number of imaginary frequencies: 0

Cartesian coordinates:

\begin{tabular}{|c|c|c|c|}
\hline & 3.691921500449 & 0.017326372449 & -2.358858614213 \\
\hline & 3.648284860418 & 0.012673070475 & -0.931817349963 \\
\hline & 6.057928182625 & 0.032125558932 & -0.885449427973 \\
\hline & 6.127499870219 & 0.036172620533 & -2.267137190890 \\
\hline & 6.926197582292 & 0.037770581985 & \\
\hline & 7.093959690956 & 0.045151534525 & -2.757123499119 \\
\hline & 1.471147711926 & -0.004695632794 & 0.532301468164 \\
\hline & 2.474460599854 & 0.000862972070 & -0.168249475879 \\
\hline & 1.298888418466 & 0.004115998448 & -3.409110064150 \\
\hline & 2.463941215501 & 0.010478180200 & -3.046462171568 \\
\hline & -1.248580194863 & -0.008945825372 & -3.713896626615 \\
\hline & -0.030167918113 & -0.002833235350 & -3.648046197655 \\
\hline & -5.370200122309 & -0.031291037456 & -2.939985413348 \\
\hline & -4.954176386575 & -0.034725960726 & -4.259390629547 \\
\hline & -2.639159540094 & -0.016012071009 & -3.497054021092 \\
\hline & -3.095291097624 & -0.011976250960 & -2.144168072038 \\
\hline & -6.409918976486 & -0.037162657589 & -2.634654757740 \\
\hline & -5.689458341119 & 25018051 & 14737 \\
\hline & -1.383673566151 & 0.052023920192 & 04219 \\
\hline & -1.831085405517 & 0.044328394875 & 334127 \\
\hline & -0.955464530357 & 0.006962254870 & 1.262612885952 \\
\hline & 0.4558 & -0.0 & 10833 \\
\hline & 0.892634759987 & 1693 & 72195 \\
\hline & 0.013165124578 & -0.053685442562 & 3.937221175888 \\
\hline $\mathrm{H}$ & -2.901930174466 & 0.092535220569 & 2.203374465320 \\
\hline $\mathrm{H}$ & 1.952796653919 & -0.093679703121 & 3.076105932540 \\
\hline & -1.564 & 0.0 & 16304 \\
\hline & -2.260917204859 & -0.000582160601 & -1.019566270802 \\
\hline $\mathrm{O}$ & 0.545949461235 & -0.108166138241 & 5.175461368376 \\
\hline $\mathrm{O}$ & -2.314378228083 & & 4.661284828109 \\
\hline $\mathrm{C}$ & 0.134686403026 & -1.19 & \\
\hline $\mathrm{H}$ & 0.616 & -1.001504377068 & 150323404 \\
\hline $\mathrm{H}$ & -0.950055633369 & -1.230547064758 & 6.159360964784 \\
\hline $\mathrm{H}$ & 0.496346866518 & -2.145174241225 & 5.628604231024 \\
\hline $\mathrm{C}$ & -2.230545773537 & 1.195860334844 & 5.616692104207 \\
\hline & -3.015 & 0.998 & 96651 \\
\hline $\mathrm{H}$ & -1.254821254061 & 1.227859974660 & 6.105113150318 \\
\hline $\mathrm{H}$ & -2.425858476652 & 2.142830312248 & 5.104200164118 \\
\hline $\mathrm{C}$ & 4.941130954907 & 0.028893257460 & -3.002800250816 \\
\hline $\mathrm{H}$ & 4.970460625496 & 0.032178492117 & -4.087635051097 \\
\hline $\mathrm{N}$ & & & -0.275388834696 \\
\hline$C$ & -3.585864059760 & -0.027199986358 & -4.535776487618 \\
\hline $\mathrm{H}$ & -3.235511435702 & -0.029999649960 & -5.562900060349 \\
\hline & -4.452401308305 & -0.020298543109 & -1.948147637440 \\
\hline $\mathrm{H}$ & -4.779034516818 & -0.019347296958 & -0.985183910540 \\
\hline & & 0.0193895099 & 0.741043018 \\
\hline
\end{tabular}


Cartesian coordinates, number of imaginary frequencies and energy for $\mathbf{7}^{\prime} \cdot \mathbf{2} \mathbf{H}$

Total energy: -1259.58637325

Number of imaginary frequencies: 0

Cartesian coordinates:

\begin{tabular}{|c|c|c|c|}
\hline & 3.676579748242 & 0.004896041710 & -2.330474445242 \\
\hline & 3.639868705592 & 0.010072082931 & -0.900457877734 \\
\hline & 6.052401830642 & 0.028962822397 & -0.879982748856 \\
\hline & 6.111608808835 & 0.023261368346 & -2.261406169424 \\
\hline & 6.926787043203 & 0.038865420066 & \\
\hline & 7.073706290727 & 0.028413509326 & -2.759644979369 \\
\hline & 1.472783991564 & 0.003062027700 & 0.572036175606 \\
\hline & 2.476525193107 & 0.003809392168 & -0.131721549122 \\
\hline & 1.285528350520 & -0.014493008315 & -3.396290049109 \\
\hline & 2.443187174263 & -0.006130089757 & -3.007830672160 \\
\hline & -1.258977977103 & -0.027699988807 & -3.743161856837 \\
\hline & -0.040377173343 & -0.022044770022 & -3.636733733253 \\
\hline & -5.390208791844 & -0.038405176424 & \\
\hline & -4.874707875290 & -0.049934844832 & 5144072 \\
\hline & -2.628216012294 & -0.030495766196 & -3.460853766388 \\
\hline & -3.114452450673 & -0.017996512687 & -2.121079433395 \\
\hline & -4.515824232369 & -0.022726699570 & -1.936415266825 \\
\hline & -6.464087803319 & -0.042088028521 & 488100 \\
\hline & -5.483094438224 & -0.062507977248 & 51150987 \\
\hline & -4.906272584501 & -0.014264557261 & 73117888 \\
\hline & -1.397846338795 & 0.082575626134 & 3.696659078819 \\
\hline & -1.835458578882 & 0660 & 10142 \\
\hline & -0.952051805635 & 444574 & 392937 \\
\hline & 0.456408025085 & 0.006008572898 & 1.546999597539 \\
\hline & 0.884674283945 & -0.02 & 2.89 \\
\hline$C$ & -0.002758916080 & 42061606 & 35731 \\
\hline & -2.904795747215 & 1123 & 0833 \\
\hline $\mathrm{H}$ & 1.943293098646 & -0.067527824289 & 3.122164811472 \\
\hline & -1.545199439015 & 1688952 & -0.010478159448 \\
\hline $\mathrm{C}$ & -2.235380641962 & -0.002253029927 & -1.017486197380 \\
\hline $\mathrm{N}$ & -3.538874381300 & -0.04576 & -4.481850278542 \\
\hline $\mathrm{O}$ & 0.509125862819 & 270542690 & 5.215667380643 \\
\hline $\mathrm{O}$ & -2.341461183625 & 0.138151276217 & 4.654576485344 \\
\hline $\mathrm{C}$ & 0.151416138993 & -1.207060305096 & 6.027463818949 \\
\hline $\mathrm{H}$ & 0.617922286483 & -1.031772449840 & 6.996361092222 \\
\hline $\mathrm{H}$ & $-0.931 \varepsilon-r-2$ & -1.29 & 6.139 \\
\hline $\mathrm{H}$ & 0.557995930166 & -2.118065795713 & 5.576863458333 \\
\hline$C$ & -2.247919482786 & 1.175716713923 & 5.665779774538 \\
\hline $\mathrm{H}$ & -3.054478301303 & 0.963359614721 & 6.366347461007 \\
\hline $\mathrm{H}$ & -1.282698223249 & & 6.173506468978 \\
\hline $\mathrm{H}$ & -2.409066666126 & 2.150468534190 & 5.195443169732 \\
\hline$c$ & 4.917584591034 & 0.011453154635 & -2.986553282159 \\
\hline $\mathrm{H}$ & 4.937683021527 & 0.007264988223 & -4.071637076521 \\
\hline $\mathrm{N}$ & 4.852930749528 & 0.022456676104 & -0.256756105826 \\
\hline & -3.169596739904 & -0.054601733689 & -5.430427332710 \\
\hline & & 0.028374943 & 0.75990170 \\
\hline
\end{tabular}

